\title{
Placido-based indices of corneal irregularity
}

D. Ramos-López, A. Martínez-Finkelshtein, G. M. Castro-Luna, D.P. Piñero and J. L. Alió

Optometry \& Vision Science

2011

This is not the published version of the paper, but a pre-print.

Please follow the link below for the final version and cite this paper as:

D. Ramos-López, A. Martínez-Finkelshtein, G. M. Castro-Luna, D.P. Piñero and J. L. Alió. Placido-based indices of corneal irregularity. Optometry \& Vision Science, Volume 88 (10), Pages 1220-1231, ISSN 1040-5488 (2011).

http://dx.doi.org/10.1097/0PX.0b013e3182279ff8 


\section{Placido-based indices of corneal irregularity}

Darío Ramos-López, B. Sc.

Department of Statistics and Applied Mathematics, University of Almería, Spain

Andrei Martínez-Finkelshtein, $\mathrm{PhD}$

Department of Statistics and Applied Mathematics, University of Almería, Spain and

Institute Carlos I of Theoretical and Computational Physics, Granada University, Spain.

Gracia M. Castro-Luna, MD, PhD

Corporación VISSUM Almería, Spain

David Piñero, $\mathrm{PhD}$

Corporación VISSUM Alicante, Spain

Jorge L. Alió, MD, PhD

Corporación VISSUM Alicante, Spain

Address all correspondence to: Darío Ramos-López, Department of Statistics and Applied Mathematics, University of Almería, 04120 Almería, Spain; e-mail: dariorl@gmail.com

This paper contains 3 Tables and 9 Figures.

Submitted on October 28, 2010 


\section{Abstract}

\section{Purpose:}

To construct a set of metrics computed from the raw keratoscopic data collected by any Placido corneal topographer. These indices that measure the irregularity of the anterior corneal surface are computed directly from the image of the mires reflected on the cornea, which is the most primary data possible. Besides the high sensitivity and specificity, this approach allows bypassing the surface or curvature reconstruction step that is currently performed by the software of any commercial Placido topographer.

\section{Methods:}

Several basic indices are proposed in order to detect irregularities on the anterior surface of the cornea, via analyzing some geometric and mathematical properties of the mires. These individual primary indices are built in a natural and intuitive way directly from the displacement of the digitized images of the rings reflected on the cornea. Additionally, compound indices are proposed (such as the generalized linear model or the classification trees) by combining some of the so-called primary indices to improve their efficiency. The computed metrics were developed and tested for the CSO topography system (CSO, Firenze,Italy), but the methodology proposed here extends easily to any other commercial Placido disks topographer.

\section{Results:}

The primary indices allow to discriminate, with excellent accuracy, between normal eyes and eyes with keratoconic corneas. Sensitivity and specificity of the primary indices is analyzed by using the ROC (receiver operating characteristic) curve methodology. Some combined indices are presented, and raise the efficiency to optimal.

\section{Conclusions:}

All the primary indices proposed exhibit very good performance at discriminating between normal and irregular corneas. The combined indices accuracy is optimal, so 
avoiding their use in clinical practice as corneal markers of disease. All these indices are fast to compute and can be easily implemented in any corneal topography system. Keywords: Corneal irregularities; keratoconus; irregularity index; diagnosis; corneal topography; Placido disks 
The measurement of corneal topography is a well established tool in corneal analysis, with a variety of clinical applications ${ }^{1-9}$. Since the early 1980 s there are commercially available topographers from many manufacturers, each with their own surface reconstruction algorithms as well as display and analysis procedures. As it is mentioned in ${ }^{10}$, "standards were not written until after the market was well developed (ANSI Z80.23-1999) and thus have not been adopted by many manufacturers who opted for consistency with their earlier models rather than the changes represented by the standards". The authors of ${ }^{10}$ reported serious inconsistency across instruments, making it cumbersome to compare maps generated from different systems, even when acquired from the same patient at the same time.

The most common technology used to measure corneal topography is the Placido disk system, where an illuminated pattern of concentric rings or mires is focused on the anterior surface of the patient's cornea and reflected back to a digital camera at the center of the cone or bowl. The images of the reflected rings are digitized along a fixed number of meridians (the number of projected rings and the number of points per ring vary from topographer to topographer, which determines the spatial resolution). This provides several thousand of points in a close-to-concentric pattern. This is the raw data that is processed by the software of the topographer to yield altitude (with respect to a surface), curvature and other parameters, using the arc-step algorithm ${ }^{11}$ or its variations. The result is typically represented as colour maps, which a priori allow subjective, qualitative analysis of the data. 
Topographic indices are an attempt to introduce objectivity into this analysis. They typically analyze either the entire corneal surface (whole cornea indices) or its specific area (regional indices) represented by a topographic map, and return a value representative of that map. Additionally, composite indices are calculated as a mathematical combination of two or more indices.

Many of the indices in use were developed as indicators for the presence or absence of keratoconus (KC). Keratoconus is an ecstatic disorder of the cornea of unknown etiology characterized by progressive thinning and cornea steepening. It is known that refractive surgery is not recommended to correct decreased vision associated with $\mathrm{KC}$. Therefore, it is important to accurately screen candidates when considering a laser refractive procedure, which stimulates further development of indices.

Additionally, topographic indices have been used in an attempt to distinguish between $\mathrm{KC}$ and other abnormalities such as contact lens-induced warpage and other forms of irregular astigmatism.

There are several very popular and widely implemented corneal indices, many of them associated with the names of Rabinowitz or Klyce and Maeda. A partial, far from complete list of primary indices is:

- Inferior-Superior or I-S index is the difference between the inferior and superior average dioptric values approximately $3 \mathrm{~mm}$ peripheral to the corneal vertex as defined by the center of the map. Inferior and superior average values are calculated by averaging 15 dioptric values on rings 
14,15 , and 16 at $30^{\circ}$ intervals in the inferior and superior portions, respectively ${ }^{3}$.

- SimK1/SimK2 are the average dioptric values of rings 8 to 10 of the major and minor axes. The greatest average power is defined as SimK1, while SimK2 is the average power of the meridian $90^{\circ}$ from the "steepest" meridian ${ }^{3}$.

- OSI or the opposite sector index reports the greatest difference in average area corrected power between opposite $45^{\circ}$ sectors, while CSI or the center/surround index reports the difference between the average area-corrected power between the central area (3- $\mathrm{mm}$ diameter) and an annulus surrounding the central area $(3-6 \mathrm{~mm})$. An analogous definition hold for CSI or the center/surround index ${ }^{7}$.

- SAl or the surface asymmetry index is the centrally weighted average of the summation of differences in corneal power between corresponding points on individual videokeratograph rings $180^{\circ}$ apart from 90 or 128 equally spaced meridians, depending on which reference one uses ${ }^{12}$.

- SRAX or the skew of steepest radial axes index is calculated according to the procedure described in ${ }^{1-4}$, see also ${ }^{10}$.

- both SRI or surface regularity index ${ }^{2}$, and the AIA or irregular astigmatism index, reported $\mathrm{in}^{3}$, have a rather complicated definition, we refer the reader to ${ }^{10}$ for explicit formulas, as well as for corrections introduced therein.

There are also popular compound or compilation indices. For instance, KISA\% is a composite index of $\mathrm{K}$ value, I-S value, $\mathrm{CYL}$, and SRAX ${ }^{4}$, while $\mathrm{KPI}$ or the 
keratoconus prediction index is based on a linear model combining DSI, OSI, CSI, SAI, SimK1, SimK2, IAI, and AA (the ratio of the interpolated data area to the area circumscribed by the last ring found in a videokeratoscope image), see $^{7}$.

However, there is no standard technique for relating corneal topographic indices from various devices, and even the same indices on different systems can give different values ${ }^{10}$. An additional concern is that most diagnostic and classification criteria for $\mathrm{KC}$ are based on anterior corneal curvature data derived from corneal topography, and for Placido-disk devices these data are secondary, i.e. computed from the digitized image of the mires reflected on the cornea. These calculations use implicit assumptions, and even their implementation in each device is considered proprietary and does not necessarily coincide with the algorithm described in literature.

The purpose of this study is to address partially this issue, proposing a methodology for building metrics based directly on the image captured by the digital camera of the Placido-based topographers. Obviously, we do not intend to mimic the arc-step method to calculate curvature from the image of the mires, and feed it as an input to one of the standard indices. Instead, our goal is to perform some intuitive and relatively simple calculations with the positions of the digitized rings in order to measure corneal irregularity. At this stage we discriminate only between "normal" and "irregular" corneas, without trying to diagnose the cause of irregularity. Nevertheless, the indices defined above 
were tested and tuned against two groups of patients, those classified as normal eyes, and those with a clinically diagnosed KC.

In what follows we will introduce two sets of primary indices, one labelled as

$\boldsymbol{P I}_{n}$ (from "Placido irregularity indices"), and the second group as ${ }^{\boldsymbol{A} \boldsymbol{R}(k)}$, from "average ring radius" of the $k$-th ring. In "Methods" we give their detailed description, as well as introduce four combined indices, based on the primary ones, built with the purpose of increasing the discriminant ability, which is assessed in "Results". This section contains also a discussion of a normalization process needed for the combined indices.

\section{METHODS}

\section{PRIMARY INDICES}

As it was mentioned in the introduction, the digitized points captured by the camera of a Placido disk corneal topographer are grouped in mires. For the sake of precision, we assume that there are 256 points equally spaced along each ring corresponding to the same number of semi-meridians (a value found in a majority of existing devices), although the indices are easily modified for any different configuration. In clinical practice, not all points are available: a number of them is missing due to digitalization errors, eye lashes obstructions, tear film disruption, and other reasons. In defining the indices we use only data from complete rings, limiting the number of rings to the maximum of 15; it should be pointed out that in the exceptional cases of really defective measurements, when the complete rings are less than 10 , the topography is 
usually discarded in clinical practice. Hence, if we denote by $N$ the number of useful innermost rings with complete data $(N \leq 15)$, we get as the input the 256 $N$ points $P_{j}$, given by their polar coordinates $\left(\rho_{j}, \theta_{j}\right), j=1, .2, \ldots, 256 N$, with $\theta_{j}=(2 \pi / 256) j \bmod 2 \pi$. Hence, if $\mathrm{M}_{\mathrm{k}}$ is the $k$-th mire $(1 \leq k \leq N)$, then $P_{j}=\left(\rho_{j}, \theta_{j}\right) \in M_{k} \Leftrightarrow j \in J_{k}:=\left\{n_{k}, n_{k}+1, \ldots, n_{k}+255\right\}, n_{k}=1+256(k-1)$. Clearly, points ${ }^{P_{j}}$ can be given also in Cartesian coordinates $\left(x_{j}, y_{j}\right)$ using the straightforward transformation $x_{j}=\rho_{j} \cos \left(\theta_{j}\right), y_{j}=\rho_{j} \sin \left(\theta_{j}\right)$.

\section{A. Indices in the Cartesian domain}

Plotted in Cartesian coordinates the digitized images of the first 15 mires look like in the Figure 1.

We fit a circle to the position of the points ${ }^{P_{j}}$ on the mire ${ }^{M_{k}}$ in the sense of the least squares (LS), using a standard procedure ${ }^{13}$. For that purpose we work with the Cartesian coordinates of ${ }^{P_{j}}$, solving by LS the overdetermined system of linear equations $A t=b$ with

$$
A=\left(\begin{array}{ccc}
x_{n_{k}} & y_{n_{k}} & 1 \\
\vdots & \vdots & \vdots \\
x_{n_{k}+255} & y_{n_{k}+255} & 1
\end{array}\right), \quad b=-\left(\begin{array}{c}
x_{n_{k}}^{2}+y_{n_{k}}^{2} \\
\vdots \\
x_{n_{k}+255}^{2}+y_{n_{k}+255}^{2}
\end{array}\right) .
$$

The solution $\mathbf{t}=\left(t_{1}, t_{2}, t_{3}\right)^{T}$ (where $\boldsymbol{t}^{T}$ denotes the matrix transpose) gives us information about the best-fit circle for the ${ }^{k}$-th mire; its center is located at $C_{k}=-\left(t_{1}, t_{2}\right)$ (in Cartesian coordinates). 
In the ideal case, e.g. when the cornea is perfectly symmetric, all centers $C_{k}$ coincide; asymmetric deformations though can yields dispersion in the location of these centers. This observation indicates that the diameter of the set of centers $C_{k}$ (normalized by the total number of rings ${ }^{N}$ ),

$$
\boldsymbol{P I}_{1}=\frac{1}{N} \max _{1 \leq n, m \leq N}\left\|C_{n}-C_{m}\right\|
$$

can be used as one of the irregularity indices. However, solely the diameter is not sufficient, as Figure 2 shows: still relatively grouped sets $\left\{C_{k}\right\}$ can exhibit an important drift of the centers, so that we control also the deviation in the consecutive ones by the following metrics:

$$
\boldsymbol{P I}_{2}=\frac{1}{N-1} \sum_{1 \leq n \leq N-1}\left\|C_{n}-C_{m}\right\|
$$

Clearly, these indices are of an isotropic character and measure the behavior of the centers of masses of each ring. If we want to capture also the spatial orientation and deformation of a mire we should fit the data with an ellipse. There are efficient methods for computation of the best-fit ellipse ${ }^{13-17}$. All of them pay a special attention to the constraint of the positive-definiteness of the underlying quadratic form (which assures that we are fitting the data with an ellipse). Taking into account the clear structure of our points and the relatively small position fluctuations, we can simplify the approach by solving the overdetermined system $\hat{A} \hat{t}=\hat{b}$, now with

$$
A=\left(\begin{array}{ccccc}
x_{n_{k}}^{2} & 2 x_{n_{k}} y_{n_{k}} & y_{n_{k}}^{2} & 2 x_{n_{k}} & 2 y_{n_{k}} \\
\vdots & \vdots & \vdots & \vdots & \vdots \\
x_{n_{k}+255}^{2} & 2 x_{n_{k}+255} y_{n_{k}+255} & y_{n_{k}+255}^{2} & 2 x_{n_{k}+255} & 2 y_{n_{k}+255}
\end{array}\right), \quad b=-\left(\begin{array}{c}
1 \\
\vdots \\
1
\end{array}\right)
$$


in the LS sense. From the solution $\hat{\mathbf{t}}=(a, b, c, d, f)^{T}$ we can find ${ }^{18}$ the axes $\left(a_{k}, b_{k}\right), a_{k} \geq b_{k}$ of the ellipse,

$$
\begin{aligned}
& \frac{a_{k}^{2}}{2}=\frac{a f^{2}+c d^{2}+b^{2}-2 b d f-a c}{\left(b^{2}-a c\right)\left(+\sqrt{(a-c)^{2}+4 b^{2}}-(a+c)\right)}, \\
& \frac{b_{k}^{2}}{2}=\frac{a f^{2}+c d^{2}+b^{2}-2 b d f-a c}{\left(b^{2}-a c\right)\left(-\sqrt{(a-c)^{2}+4 b^{2}}-(a+c)\right)},
\end{aligned}
$$

not necessarily collinear with the $(x, y)$ axes.

We can measure the irregularity of the images of the mires by studying the dispersion of the values of the axis ratios $r_{k}=a_{k} / b_{k} \geq 1$ of the $k$-th ellipse (see Figure 3, left):

$$
\mathbf{P I}_{3}=\sqrt{\frac{1}{N} \sum_{1 \leq k \leq N}\left(r_{k}-\bar{r}\right)^{2}}, \quad \bar{r}=\frac{1}{N} \sum_{1 \leq k \leq N} r_{k} .
$$

\section{B. Indices in the polar domain}

Now we use the polar coordinates $\left(\rho_{j}, \theta_{j}\right)$, of each ${ }^{P_{j}}$, considering $\rho$ as function of $\theta$. Plotted in polar coordinates the digitized images of the first 15 mires look like in the Figure 4.

Let

$$
\mathbf{A R}(k)=-\bar{\rho}_{k}=-\frac{1}{256} \sum_{j \in J_{k}} \rho_{j}, \quad k=1, \ldots, \min \{10, N\},
$$

and $R_{k}=\max _{j \in J_{k}}\left|\rho_{j}-\bar{\rho}_{k}\right|, k=1, \ldots, N ;$ then index

$$
\boldsymbol{P I}_{4}=\max _{1 \leq k \leq N} R_{k},
$$


is correlated with ${ }^{P I_{3}}$ introduced above, and measures again how well points ${ }_{j}$ fit the circular pattern (see Figure 3, right). A N-S and E-W asymmetry, characteristic in many forms of KC, can be detected by analyzing the values of

$$
\begin{gathered}
\mathbf{P I}_{5}=\frac{1}{N}\left|\sum_{0<\theta_{j}<\pi} \rho_{j}-\sum_{\pi<\theta_{j}<2 \pi} \rho_{j}\right| \\
\mathbf{P I}_{6}=\frac{1}{N}\left|\sum_{\frac{\pi}{2}<\theta_{j}<\frac{3 \pi}{2}} \rho_{j}-\sum_{0 \leq \theta_{j}<\frac{\pi}{2} o r \frac{3 \pi}{2}<\theta_{j}<2 \pi} \rho_{j}\right| .
\end{gathered}
$$

For a more sensitive analysis we compute in the $\theta-\rho_{\text {domain the linear }}$ regression for the points corresponding to each mire, $\rho=m_{k} \theta+\beta_{k} k=1, \ldots, N$. Slopes ${ }^{m_{k}}$ different from zero are an indication of the upper-lower asymmetry. In practice, they take rather small values, with predominance of negative slopes (corresponding to the more common protrusion in $\mathrm{KC}$ in the lower part of the cornea). Recall that the way the corneal topographers order the points, lower values of $\theta$ correspond to the upper half of the disk. Hence, index

$$
\mathbf{P I}_{7}=\max _{k}\left|m_{k}\right|,
$$

measures the slope of the steepest regression line.

The indices defined above analyze the global asymmetry of the mires. However, diseases such as KC are characterized by a localized steepening of the cornea. Values based on linear regression cannot help, and we use additional indices of a local character, inspired by the iterative algorithm introduced in ${ }^{19-20}$ that fits the data by anisotropic radial basis Gaussian functions (A-RBGF). We use here only one iteration of its simplified version, based on the 
purely radial basis functions, applied to the data $\left(\rho_{j}-\bar{\rho}_{k}, \theta_{j}\right), j \in J_{k}$, $\bar{\rho}_{k}=\frac{1}{256} \sum_{j \in J_{k}} \rho_{j}$, which for each $k=1, \ldots, N_{\text {yields an approximation }} \rho_{j} \approx \rho\left(\theta_{j}\right)$ with

$$
\rho=\bar{\rho}_{k}+c_{k} e^{-\alpha_{k}\left|\eta_{k}-\theta\right|^{2}}
$$

Briefly, $\eta_{k}$ is picked among the angles $\theta_{j}, j \in J_{k}$, corresponding to $\max _{j \in J_{k}}\left|\rho_{j}-\bar{\rho}_{k}\right|$; with $\eta_{k}$ fixed we consider an overdetermined system of linear equations, that we denote again by $A t=b$, with

$$
A=\left(\begin{array}{cc}
1 & -\left|\eta_{k}-\theta_{j_{1}}\right|^{2} \\
\vdots & \vdots \\
1 & -\left|\eta_{k}-\theta_{j_{k}}\right|^{2}
\end{array}\right) \quad b=\left(\begin{array}{c}
\log \left(\rho_{j_{1}}-\bar{\rho}_{k} \mid\right) \\
\vdots \\
\log \left(\left|\rho_{j_{k}}-\bar{\rho}_{k}\right|\right)
\end{array}\right)
$$

The indices $j=j_{1}, \ldots, j_{k}$ are those for which $\left|\eta_{k}-\theta_{j}\right|^{2}<0.05$ (corresponding approximately to 10 nearest neighbours along the mire) and $\rho_{j}-\bar{\rho}_{k}$ match the sign of this value at $\eta_{k}$. The shape parameter is $\alpha_{k}=t_{2}$, where $t=\left(t_{1}, t_{2}\right)^{T}$ is the solution of this system in the sense of the least squares. Finally, the scaling factor $c_{k}$ is computed fitting again equation (1) in the sense of linear LS to the same data. Using these definitions we introduce several indices. A large value of the $\ell^{2}$ norm of the vector of scaling coefficients,

$$
\boldsymbol{P I}_{8}=\sum_{1 \leq k \leq N} c_{k}^{2}
$$

could indicate global irregularities on all the mires. Still, large ${ }^{c_{k}}$ 's could correspond also to large values of $\alpha_{k}$ 's (high and steep bell-shaped curves). 
Since the area under the graph of the normalized Gaussian $y=c \pi^{-1 / 2} \exp \left(-\alpha x^{2}\right)$, $c>0$, is equal to $c / \sqrt{\alpha}$, we define the values

$$
A_{k}=\frac{\left|c_{k}\right|}{\sqrt{\alpha_{k}}}, \quad k=1, \ldots, N
$$

and two more indices,

$$
\begin{gathered}
\boldsymbol{P I}_{9}=\max _{1 \leq k \leq N} A_{k}, \\
\boldsymbol{P I}_{10}=\frac{1}{N-2} \sum_{k} A_{\sigma(k)}^{2} .
\end{gathered}
$$

The summation in the last index goes along all $k=1, \ldots, N$, excluding two extreme (smallest and largest) values of $A_{k}$, for the sake of robustness with respect to the measurement errors typically present at the outermost rings (see Figure 5).

The distribution of the centers $\eta_{k}$ in (1) bears additional information: a cluster of these centers might indicate a localized distortion of the adjacent rings (see Figure 6). Hence, we define

$$
\boldsymbol{P I}_{11}=\frac{1}{N^{2}} \sum_{1 \leq k, m \leq N}\left|\eta_{k}-\eta_{m}\right|^{2}
$$

\section{COMBINED INDICES}

As we will see in the next Section, neither index defined in Section I works as a perfect binary classifier for KC. However, we can create a synergy using their combination. There are several approaches for defining combined indices. In this paper we explore two of them. The result of the first one is a continuous value that can be interpreted as a probability of $\mathrm{KC}$ or other corneal disorder, and can be used as a degree of corneal irregularity. The second one gives us 
binary variables that render 0 or 1 meaning a "normal" or "irregular" cornea, respectively, that do not provide though the degree of irregularity. All these indices are easily computed once the corresponding coefficients and thresholds are found. For this analysis, again the statistical tool $R^{21}$ was used; for the sake of reproducibility, we will make reference to the $\mathrm{R}$ functions and toolboxes.

\section{A. A generalized linear model}

A generalized linear model (GLM) is an extension of the ordinary least squares regression; it allows the linear model to be related to the response variable via a link function and by allowing the magnitude of the variance of each measurement to be a function of its predicted value ${ }^{22-23}$. In this work, we use the well-known "probit" link function that is the inverse of the cumulative distribution function of the standard normal distribution $N(0,1)$; it is a strictly increasing function on the real axis taking values in [0; 1], with no explicit algebraic expression. The R function $\mathrm{glm}$ from the R package stats was applied to the database of 32 normal and 20 keratoconic corneas mentioned in Section III below. As an output, we obtained a GLM model that was adjusted a posteriori in order to get more reasonable values of coefficients. The described procedure yields a combined index that we call GLPI (from Generalized Linear Placido Irregularity index), calculated from the primary indices as follows:

$$
\boldsymbol{G L P I}=100 \times \operatorname{probit}(\eta)
$$

with

$$
\eta=10^{-2}\left(-784.5+0.19 \times \boldsymbol{P I}_{2}+5.5 \times \boldsymbol{P I}_{3}+2 \times \boldsymbol{P I}_{5}+4.1 \times \boldsymbol{P I}_{6}+4 \times \boldsymbol{A R}(4)\right) .
$$

For this calculation we use the normalized values of the primary indices, as explained in Section IV. In this case, the linear predictor $\eta$ takes its minimum 
value -7.845 when all indices are equal to 0 , and its maximum value 15.84 when all the indices are equal to 150 . Index $\boldsymbol{G L P I}$ can be considered a measure of the corneal irregularity or a probability (in \%) of a corneal disease.

\section{B. Classification Trees}

Classification trees are simple to use and well established binary classifiers; they can be constructed by means of the $\mathrm{R}$ function rpart from the $\mathrm{R}$ package of the same name. Applying it to the database mentioned before, the following three best-performing classification trees were built (with a 100\% of correct classifications), listed here in increasing order of complexity:

Tree 1 (CT1): in this simplest case, the cornea is considered irregular if the following conditions are verified:

$$
\boldsymbol{P I}_{2} \geq 50 \text { and } \quad \boldsymbol{P \boldsymbol { I } _ { 1 1 }} \geq 15.81 .
$$

Otherwise the cornea is classified as normal (Figure 8, left). The philosophy underlying this tree is that any cornea for which $\boldsymbol{P I}_{2}$ is below the critical value 50 (according to the normalization explained in Section IV) is automatically classified as normal. In the case it does not comply with this condition, normality is still certified if the companion index, $\boldsymbol{P I}_{11}$, is considerably small $(<15.18)$.

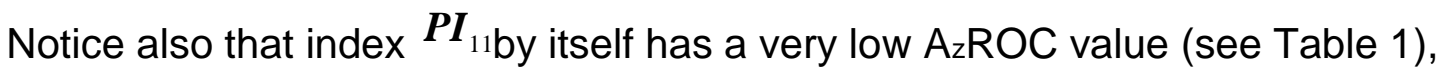
but serves to raise the discrimination capacity of ${ }^{P I_{2}}$.

Tree $2\left(\mathrm{CT}_{2}\right)$ : the cornea is considered normal if the following conditions hold:

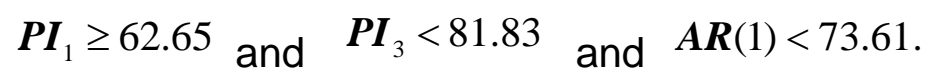


Otherwise the cornea is classified as irregular (Figure 8, right). Observe that for this classification tree, a conjunction of three rather conservative conditions (the cut-off values are considerably greater than 50 ) yields a validation of the cornea as normal.

Tree 3 (CT): the cornea is classified as normal if $\boldsymbol{P I}_{2}<46.29$ or, if this condition fails, if either $\boldsymbol{A R}(1)<50.54$ or $\boldsymbol{P I}_{4}<24.44$. Otherwise the cornea is considered irregular (Figure 9). A comparison with $\boldsymbol{C} \boldsymbol{T}_{1}$ shows that if a more restrictive

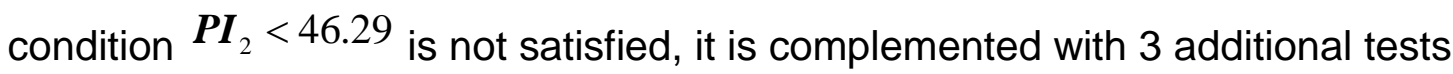
to achieve the desired accuracy.

\section{RESULTS}

\section{ASSESSMENT OF THE PRIMARY INDICES}

In this Section we analyze the performance of the primary indices introduced above. For that purpose we use two test groups of Placido disks images, one corresponding to 32 normal eyes and another of 20 keratoconic eyes, from patients ranging in age from 2 to 74 year old (mean age of 32 , standard deviation of 14.6 years). The inclusion in the $\mathrm{KC}$ group was based on the standard criteria for the diagnosis of this corneal condition and the absence of any previous surgical intervention that could have altered the corneal properties. The following signs were considered at diagnosis ${ }^{24}$ : corneal topography revealing an asymmetric bowtie pattern with or without skewed axes and at least one keratoconus sign on slit-lamp examination, such as stromal 
thinning, conical protrusion of the cornea at the apex, Fleischer ring, Vogt striae or anterior stromal scar. In those patients wearing contact lenses for the correction of the refractive error, only data obtained after an appropriate contact lens discontinuation were considered: at least two weeks for soft contact lenses and at least four weeks for rigid gas permeable contact lenses. The exclusion criteria for the keratoconus group were other ocular active pathology at the moment of diagnosis and the presence of an advanced keratoconus (grade 4 according to the Alió-Shabayek grading system ${ }^{25}$ ). The group of normal eyes only included eyes with no other ocular pathology, previous ocular surgery or irregular corneal pattern.

All patients were informed about the study and signed an informed consent document in accordance with the Helsinki Declaration.

A particular index from the set of indices defined in Section I, let us call it $I$, takes values $I_{1}^{N}, \ldots, I_{32}^{N}$ corresponding to the normal eyes, and values $I_{1}^{K}, \ldots, I_{20}^{K}$, corresponding to eyes with KC. In the way these indices were defined, we can expect that the values for normal eyes are in general smaller than those for the keratoconic ones (this explains the negative sign in some of the indices).

The receiver operating characteristic $(\mathrm{ROC})$ curve analysis is a well established tool for assessing the discriminant capability of a model. For that purpose, for every cut-off or threshold value $I^{(0)}$ we define 


$$
F P=\#\left\{j: I_{j}^{N} \geq I^{(0)}\right\}, \quad F N=\#\left\{j: I_{j}^{K}<I^{(0)}\right\},
$$

where $F P$ and $F N$ stand for "false positive" and "false negative", respectively (\#A stands for the cardinality or number of elements in the set $A$ ). It is convenient also to introduce the number of "true positive" and "true negative" cases, $T N=32-F P$ and $T P=20-F N$. Normalizing these values, we get the fraction or rate of false positive and negatives, and of true positives and negatives:

$$
\begin{aligned}
& F P R=\frac{F P}{32}, \\
& F N R=\frac{F N}{20}, \quad T P R=\frac{T N}{32}=1-F P R,
\end{aligned}
$$

These fractions, computed for each threshold value $I^{(0)}$, allow us to plot the ROC curves for all indices introduced above. A ROC curve represents graphically the "sensitivity", or $T P R$, vs. (1- "specificity"), or $F P R$, as $I^{(0)}$ is varied. Since the number of cases is finite, the ROC curve takes a piecewise constant form, see Figure 7. A standard criterion for assessing the performance of an index is the area under the ROC curve $\left(A_{z} R O C\right)$ : the higher it is, the better, being area 1 the ideal maximum. The third row of Table 1 shows the values of $A_{z} R O C$ for the described primary indices. We see that the values for the best five are not less than 0.95, being optimal the indices $\boldsymbol{P I _ { 1 }}$ and $\boldsymbol{P I _ { 2 }}$. Along with the $\mathrm{A}_{z} \mathrm{ROC}$ method we have considered also an alternative way to select the best index, based on the consideration that for a perfect binary classifier there exists a threshold value $I^{(0)}$ for which both $T N R=1$ and $T P R=1$. Hence, we can consider a better classifier the index for which

$$
\max _{I^{(0)}} \frac{T N R+T P R}{2}
$$


is greater. For comparison, the second row of Table 1 shows the values of the maximum average of $T N R$ and $T P R$ for each index. We see that again the optimal indices are $\boldsymbol{P \boldsymbol { I } _ { 1 }}$ and $\boldsymbol{P I}_{2}$, and that both used criteria give consistent results.

Practically all calculations were carried with Matlab (The MathWorks, Inc., Natick, MA) using a custom written software. For the ROC analysis, the version 2.10.1 of the freely available statistical tool $R^{21}$ was used.

\section{NORMALIZATION OF THE PRIMARY INDICES}

Since the metrics defined in Section I have very different units and scales, in order to build a combined index or a decision tree we need to find a proper normalization of each index to make them suitable for further joint analysis.

In the way the indices have been defined, $I_{\min }=\min \left\{I_{j}^{N}: j=1, \ldots, 32\right\}<I^{*}$, where $I^{*}$ is the threshold value $I^{(0)}$ maximizing (2). In order to get the modified index $\hat{J}$ corresponding to the primary index I we perform the linear transformation

$$
\hat{J}=a I+b,
$$

which maps the interval $\left[{ }_{\min }, I^{*}\right]$ onto $[0,50]$. In this way, all values of $J$ accounted in $T N$ are between 0 and 50 . In order to avoid negative and very large positive values we define finally the normalized index

$$
J=\left\{\begin{array}{ccc}
0, & \text { if } & \hat{J} \leq 0 \\
150, & \text { if } & \hat{J} \geq 150 \\
\hat{J} & \text { otherwise. } &
\end{array}\right.
$$


In this way, we consider every value of $J \leq 50$ as normal, as well as all values above this threshold as indicating an irregularity. The values of the coefficients $a$ and $b$ in (3) corresponding to each primary index introduced above appear in Table 2. In the next Section we only use the values of the normalized indices $\boldsymbol{P I}_{n}$ and $^{\boldsymbol{A R}(k)}$, obtained after applying (3) with the values from Table 2. In order to simplify notation, we omit in what follows the "hat" when referring to these indices.

\section{DISCUSSION}

It is known ${ }^{26}$ that data obtained from the first corneal surface can be used as a highly sensitive and specific diagnostic tool for the early detection of ecstatic diseases such as the subclinical KC.

By means of standard statistical methods, metrics were constructed that differentiated, with excellent accuracy, between normal eyes and eyes with irregular corneas. They are based on a set of individual corneal irregularity indices built in a natural and intuitive fashion directly from the digitized images of the Placido rings reflected on the cornea. Although the indices were developed and tested for the CSO topography system (CSO, Firenze, Italy), the methodology proposed here extends easily to any other commercial Placido topographer. Future studies including a larger sample size drawn a priori will allow further improvement of detection and classification results, especially for subclinical $\mathrm{KC}$, which is the most difficult entity to detect. It is known that the 
importance of an early detection of such cases lays in avoiding undergoing excimer laser refractive surgery procedures in these weakened and altered corneas, and also in selecting the most appropriate treatment option (intracorneal ring segments, crosslinking, contact lens. . . ) in order to prevent $\mathrm{KC}$ progression.

Our indices not necessarily supersede or supplant the common metrics such as $\mathrm{KISA} \%$ or KPI, but can serve as a valuable complements in clinical practice. In our experiments we have found data of real patients, clinically diagnosed with $\mathrm{KC}$ but with rather low $\mathrm{KPI}$, and for which our indices gave excellent results, see Table 3 for an example. They present the advantage of reliability with respect to existing indices that are based on proprietary or questionable algorithms of derivation of curvature and corneal power from the raw ring images. Additionally, they present the advantage of simplicity with respect to more sophisticated approaches, such as neural networks ${ }^{27-28}$. See ${ }^{29}$ for a preliminary report on the first assessment of the applicability of these indices to the detection of $\mathrm{KC}$.

Undoubtedly, the topography of the posterior surface and the thickness of the cornea have an additional relevant information that can help in the early detection of $\mathrm{KC}$ and other ecstatic diseases ${ }^{30-34}$. However, nowadays this information is collected only by more sophisticated devices based on the Scheimpflug imaging, the optical coherence tomography and similar alternatives, which are still not widely available in the clinical practice. In this 
sense, the simple and easy to use Placido disk topographers represent a vast majority of devices. It is known also that they are especially precise for measuring the central part of the cornea.

As it follows from the results in Table 1, all individual indices have $A_{z} R O C$ values from good to excellent, even in comparison with existing primary metrics; similar conclusions are obtained for the sensitivity and specificity ${ }^{7}$. However, the use of combined indices, especially the generalized linear model or the classification trees, allows raising the efficiency to optimal. These metrics have a rather different character. The generalized linear model gives a continuum of values that can be used not only to detect an irregularity but also to assess its degree; however, values that fall close to the cut-off value (30 in our case) should be examined with care. The classification trees have the advantages of the simplicity of application and the binary outcome ("irregularity yes or not"), so can be used as a warning for a clinician for a further analysis.

At this stage, the indices developed above are of a general character and do not intend to differentiate between types of ecstasies or degrees of KC. Rather they serve as a reliable detector of existence of a corneal irregularity, as an aid for a clinician. However, the ability to discriminate between several types of diseases of some of these indices or to assign a meaning to the value of each metrics, especially of the generalized linear model $\boldsymbol{G L P I}$, is currently under study. We also plan to extend this methodology to metrics for both anterior and posterior faces of the corneas, using the raw data collected by more specialized topographers, such as those based on the Scheimpflug imaging. Besides their diagnostic value, these metrics would allow for an indirect study of the 
biomechanical properties of the affected corneas. We hope that the approach proposed here will shed a new light on the diagnosis of ecstatic diseases, opening new perspectives to be analyzed in the future research.

\section{ACKNOWLEDGMENTS}

A.M.-F. is supported in part by Junta de Andalucía grants FQM-229 and P09FQM-4643, and by the Ministry of Science and Innovation of Spain (project code MTM2008-06689-C02-01). Both A.M.-F. and D.R.-L. are also supported in part by Junta de Andalucía grant P06-FQM-01735. G. C.-L., D. P. and J. L. A. acknowledge the support of the Spanish grant FIS PI08/90519.

\section{REFERENCES}

[1] Y. S. Rabinowitz, Journal of Refractive Surgery, 11, 371 (1995).

[2] S. E. Wilson and S. D. Klyce, Arch. Ophtalmol., 109, 349 (1991).

[3] N. Maeda, S. D. Klyce, and M. K. Smolek, Archives of Ophthalmology, 113, 870 (1995).

[4] Y. S. Rabinowitz and K. Rasheed, J. Cataract Refract. Surg., 25, 1327 (1999).

[5] Y. S. Rabinowitz and P. J. McDonnell, Refractive and Corneal Surgery, 5, 400 (1989).

[6] M. K. Smolek and S. D. Klyce, Invest. Ophthalmol. Vis. Sci., 38, 2290 (1997).

[7] N. Maeda, S. D. Klyce, M. K. Smolek, and H. W. Thompson, Invest. Ophthalmol. Vis. Sci., 35, 2749 (1994). 
[8] N. Maeda, S. D. Klyce, and M. K. Smolek, Invest. Ophthalmol. Vis. Sci., 36, 1327 (1995).

[9] L. J. Maguire and W. M. Bourne, American J. Ophthalmology, 108, 107 (1989).

[10] A. M. Mahmoud, C. Roberts, R. Lembach, E. E. Herderick, T. T. McMahon, and T.

C. S. Group, Optometry and Vision Science, 83, 682 (2006).

[11] C. Campbell, Optometry and Vision Science, 74, 899 (1997).

[12] S. A. Dingeldein, S. D. Klyce, and S. E. Wilson, Refractive and Corneal Surgery, 5, 372 (1989).

[13] S. J. Ahn, W. Rauh, and H.-J. Warnecke, Pattern Recognition, 34, 2283 (2001).

[14] K. F. Mulchronea and K. R. Choudhury, Journal of Structural Geology, 26, 143 (2004).

[15] A. W. Fitzgibbon, M. Pilu, and R. B. Fisher, IEEE Transactions on Pattern Analysis and Machine Intelligence, 21, 476 (1999).

[16] D. Hart and A. J. Rudman, Computers \& Geosciences, 23, 189 (1997).

[17] R. Halir and J. Flusser, "Numerically stable direct least squares fitting of ellipses," (1998).

[18] E. W. Weisstein, "Ellipse. From MathWorld-A Wolfram web resource," http://mathworld.wolfram.com/Ellipse.html.

[19] A. Martínez-Finkelshtein, D. Ramos-López, G. M. Castro-Luna, and J. L. Alió, Invest. Ophthalmol.

Vis. Sci., 51, ARVO E-Abstract 5690 (2010).

[20] A. Martínez-Finkelshtein, D. Ramos-López, G. M. Castro-Luna, and J. L. Alió, “An adaptive algorithm for the cornea modeling from keratometric data," (2010), preprint. [21] R Development Core Team, R: A language and environment for statistical computing, R Foundation for Statistical Computing, Vienna, Austria (2009). [22] P. McCullagh and J. A. Nelder, Generalized Linear Models, 2nd ed. (Chapman and Hall/CRC, 1989).

[23] J. F. Faraway, Linear Models with R (Chapman and Hall/CRC, 2005). 
[24] Y. S. Rabinowitz, Surv. Ophthalmol., 42, 297 (1998).

[25] J. L. Alió and M. H. Shabayek, Journal of Refractive Surgery, 22, 539 (2006).

[26] J. Bühren, C. Kühne, and T. Kohnen, American J. Ophthalmology, 143, 381 (2007).

[27] P. A. Accardo and S. Pensiero, J. Biomed. Inform., 35, 151 (2002).

[28] L. A. Carvalho, Optometry and Vision Science, 82, 151 (2005).

[29] D. Piñero, A. Martínez-Finkelshtein, D. Ramos-López, G. M. Castro-Luna, and J.

L. Alió, "Anterior corneal irregularity characterization by means of new Placido-based indices: a preliminary study," (2010), submitted.

[30] T. Tomidokoro, A.and Oshika, S. Amano, S. Higaki, N. Maeda, and K. Miyata, Ophthalmology, 107, 1328 (2000).

[31] S. Quisling, S. Sjoberg, B. Zimmerman, K. Goins, and J. Sutphin, Ophthalmology, 113, 1629 (2006).

[32] Z. Schlegel, T. Hoang-Xuan, and D. Gatinel, J. Cataract Refract. Surg., 34, 789 (2008).

[33] U. de Sanctis, C. Loiacono, L. Richiardi, D. Turco, B. Mutani, and F. M. Grignolo, Ophthalmology, 115, 1534 (2008).

[34] J. Bühren, D. Kook, G. Yoon, and T. Kohnen, Invest. Ophthalmol. Vis. Sci., 51, 3424 (2010). 


\section{FIGURE LEGENDS}

Figure 1. Representation in Cartesian coordinates of the digitized points ${ }^{P_{j}}$ for a normal cornea (left) and in a case of keratoconus (right).

Figure 2. Relative positions of centers $C_{k}$ for a normal cornea and two cases of keratoconus.

Figure 3. Left: values of the ratios $r_{k}=a_{k} / b_{k} \geq 1$ on each ring $1 \leq k \leq 15$ for a normal and a keratoconic corneas; dotted lines indicate $\bar{r}$ in each case. Right: values of $R_{k}$ for a normal and a keratoconic corneas.

Figure 4. Representation in polar coordinates of the digitized images of the first 15 mires for a normal cornea (left) and in a case of keratoconus (right).

Figure 5. Coefficients $c_{k}$ (left) and areas $A_{k}$ (right) for a normal and a keratoconic corneas. 
Figure 6. Location of centers $\eta_{k}$ for a normal cornea (left) and in a case of keratoconus (right).

Figure 7. Receiver Operating Characteristic (ROC) curves for the best four primary irregularity indices: ${ }^{\mathbf{P I}} \mathbf{I}_{1}$ (upper left), ${ }^{\mathbf{P I}}{ }_{2}$ (upper right), ${ }^{\mathbf{P I}_{4}}$ (lower left) and $\mathbf{P I}_{8}$ (lower right).

Figure 8. Classification Trees CT1 and CT2.

Figure 9. Classification Tree CT3. 
TABLE 1. ROC (Receiver Operating Characteristic) efficiency criteria for the best performance primary indices (sorted in a decreasing $\mathrm{A}_{2} \mathrm{ROC}$ value order).

\begin{tabular}{|c|c|c|c|c|c|c|c|c|c|c|c|c|c|}
\hline Index & $\boldsymbol{P I}_{2}$ & $\boldsymbol{P I}_{1}$ & $\boldsymbol{P I}_{8}$ & $\boldsymbol{P I}_{4}$ & $\boldsymbol{P I}_{10}$ & $\boldsymbol{P I}_{9}$ & $\boldsymbol{P I}_{3}$ & $\boldsymbol{P I}_{5}$ & $\boldsymbol{P I}_{7}$ & $\boldsymbol{P I}_{6}$ & $\boldsymbol{A R}(4)$ & $\boldsymbol{A R}(1)$ & $\boldsymbol{P I}_{11}$ \\
\hline $\max \frac{T N R+T P R}{2}$ & 0.97 & 0.93 & 0.89 & 0.88 & 0.88 & 0.86 & 0.86 & 0.89 & 0.86 & 0.72 & 0.75 & 0.72 & 0.65 \\
\hline $\mathrm{A}_{2} \mathrm{ROC}$ & 0.98 & 0.98 & 0.96 & 0.95 & 0.95 & 0.93 & 0.91 & 0.90 & 0.83 & 0.77 & 0.71 & 0.71 & 0.66 \\
\hline
\end{tabular}


TABLE 2. Primary indices normalization constants for the linear scaling defined in (3).

\begin{tabular}{|c|c|c|c|c|c|}
\hline Index name & $\mathrm{a}$ & $\mathrm{b}$ & Index name & $\mathrm{a}$ & $\mathrm{b}$ \\
\hline $\boldsymbol{P I _ { 1 }}$ & $1.24 \times 10^{4}$ & $-1.25 \times 10^{-7}$ & $\boldsymbol{A R}(1)$ & $1.96 \times 10^{3}$ & $4.45 \times 10^{2}$ \\
\hline $\boldsymbol{P I}_{2}$ & $9.70 \times 10^{3}$ & $-1.83 \times 10^{1}$ & $\boldsymbol{A R}(2)$ & $5.63 \times 10^{2}$ & $2.80 \times 10^{2}$ \\
\hline $\boldsymbol{P I}_{3}$ & $5.24 \times 10^{3}$ & $-1.38 \times 10^{1}$ & $\boldsymbol{A R}(3)$ & $4.87 \times 10^{2}$ & $3.31 \times 10^{2}$ \\
\hline $\boldsymbol{P I}_{4}$ & $4.43 \times 10^{2}$ & $-1.32 \times 10^{1}$ & $\boldsymbol{A R}(4)$ & $3.18 \times 10^{2}$ & $2.98 \times 10^{2}$ \\
\hline $\boldsymbol{P I}_{5}$ & $2.13 \times 10^{3}$ & -0.07 & $\boldsymbol{A R}(5)$ & $2.88 \times 10^{2}$ & $3.21 \times 10^{2}$ \\
\hline $\boldsymbol{P I}_{6}$ & $2.25 \times 10^{3}$ & -1.4 & $\boldsymbol{A R}(6)$ & $2.23 \times 10^{2}$ & $3.04 \times 10^{2}$ \\
\hline $\boldsymbol{P I}_{7}$ & $2.88 \times 10^{3}$ & $-7.33 \times 10^{-4}$ & $\boldsymbol{A R}(7)$ & $2.41 \times 10^{2}$ & $3.69 \times 10^{2}$ \\
\hline $\boldsymbol{P I}_{8}$ & $2.05 \times 10^{2}$ & $-1.42 \times 10^{1}$ & $\boldsymbol{A R}(8)$ & $1.83 \times 10^{2}$ & $3.25 \times 10^{2}$ \\
\hline $\boldsymbol{P I}_{9}$ & $6.20 \times 10^{2}$ & -7.66 & $\boldsymbol{A R}(9)$ & $1.88 \times 10^{2}$ & $3.67 \times 10^{2}$ \\
\hline $\boldsymbol{P I}_{10}$ & $2.32 \times 10^{4}$ & -1.03 & $\boldsymbol{A R}(10)$ & $2.07 \times 10^{2}$ & $4.47 \times 10^{2}$ \\
\hline $\boldsymbol{P I}_{11}$ & 4.4 & 55.66 & & & \\
\hline
\end{tabular}


TABLE 3. Values of the indices for an actual patient diagnosed with $\mathrm{KC}$, but with $\mathrm{KPI}=16$. Recall that the cornea is considered irregular for $\mathrm{KPI}$ values equal or above 50. Additionally, all classification trees $\mathbf{C T}$ (classification tree $\mathrm{n}$ ) assessed the cornea as irregular, while the probability of a corneal irregularity by the GLPI (Generalized Linear Placido Irregularity index) was of $93 \%$.

\begin{tabular}{|c|c|c|c|c|c|c|c|c|c|c|c|c|c|}
\hline Index & $\boldsymbol{P I}_{1}$ & $\boldsymbol{P I}_{2}$ & $\boldsymbol{P I}_{3}$ & $\boldsymbol{P I}_{4}$ & $\boldsymbol{P I}_{5}$ & $\boldsymbol{P I}_{6}$ & $\boldsymbol{P I}_{7}$ & $\boldsymbol{P I}_{8}$ & $\boldsymbol{P I}_{9}$ & $\boldsymbol{P I}_{10}$ & $\boldsymbol{P I}_{11}$ & $\boldsymbol{A R}(1)$ & $\boldsymbol{A R}(4)$ \\
\hline Value & 78 & 61 & 37 & 61 & 150 & 40 & 3 & 67 & 70 & 89 & 55 & 64 & 38 \\
\hline
\end{tabular}




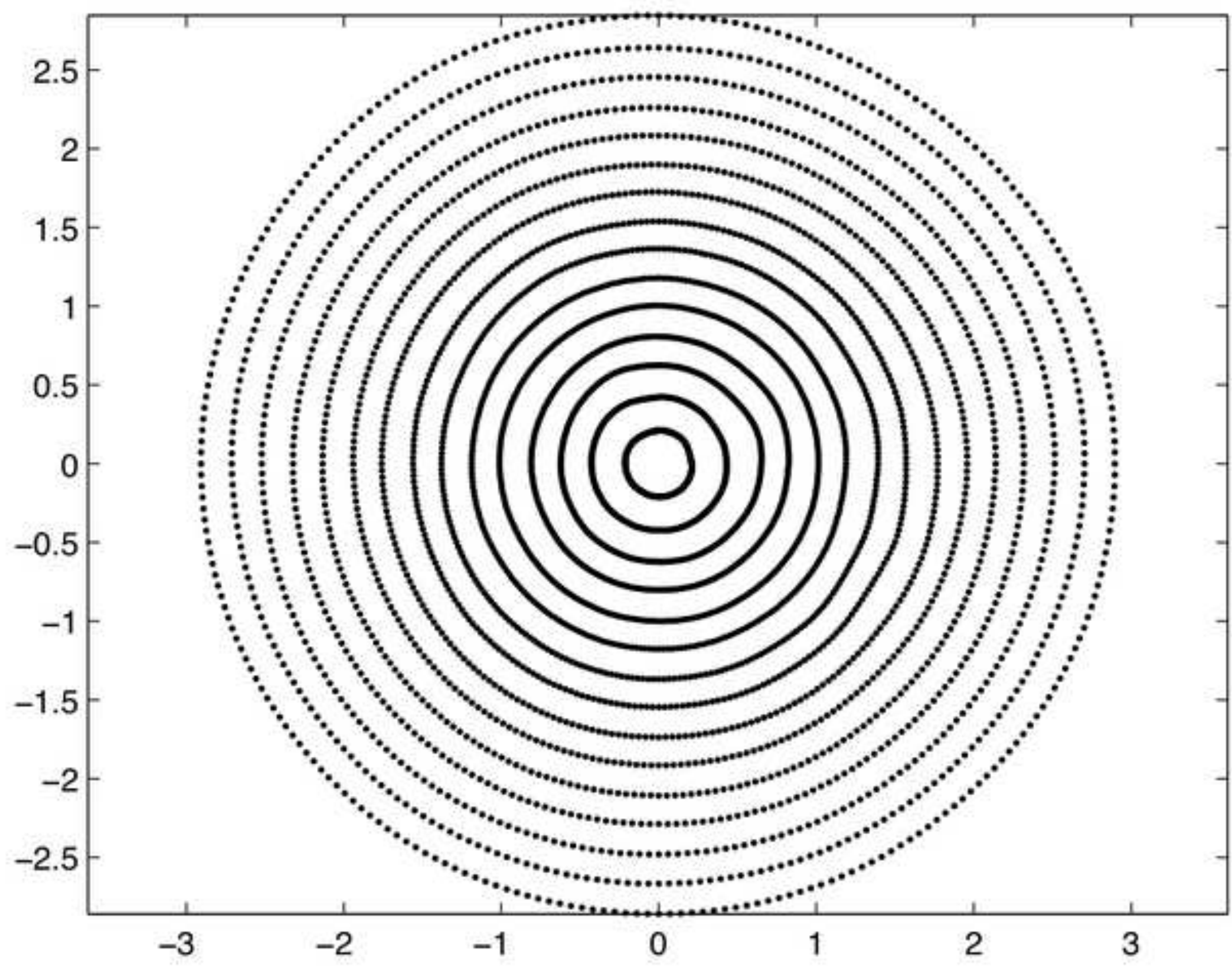




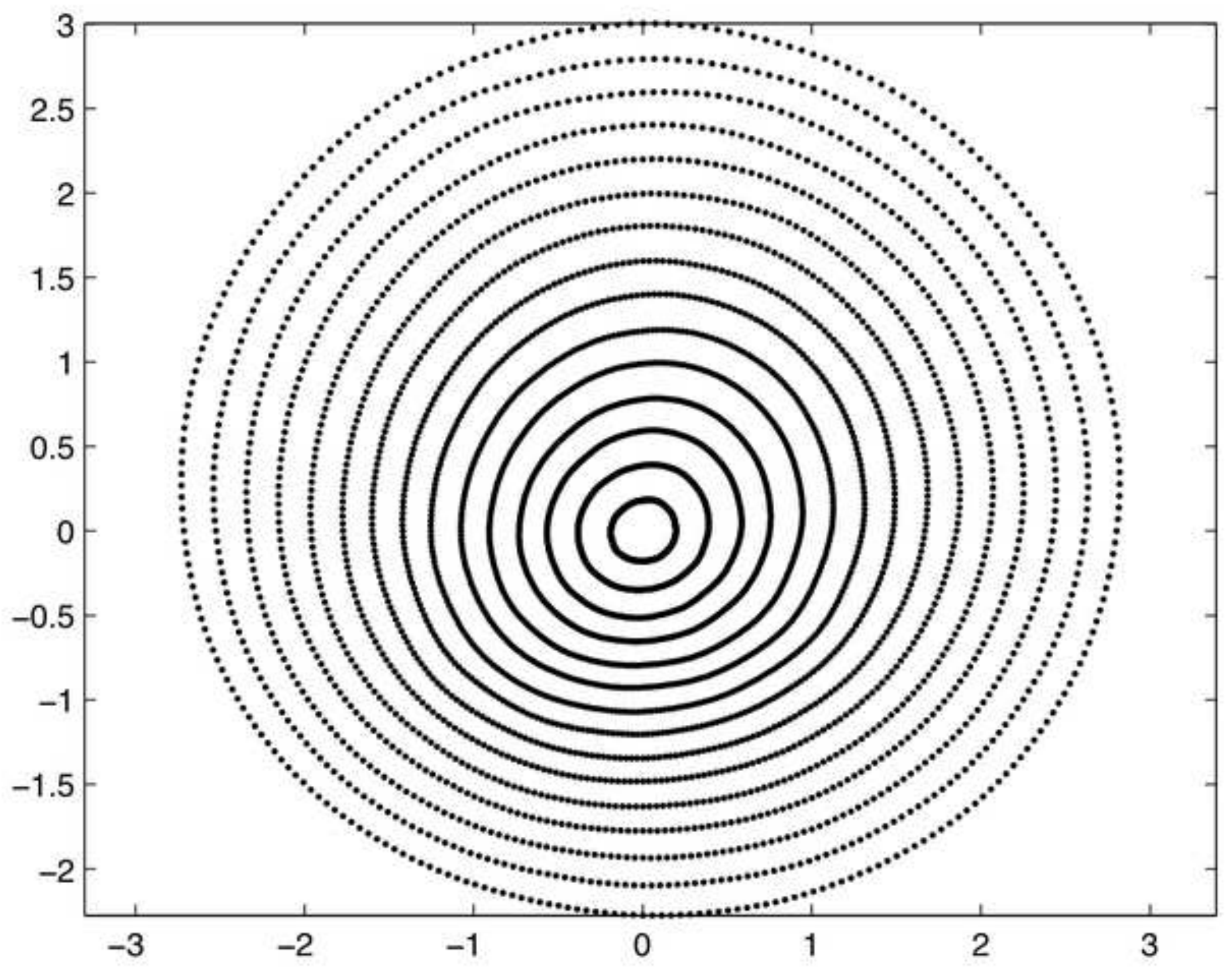




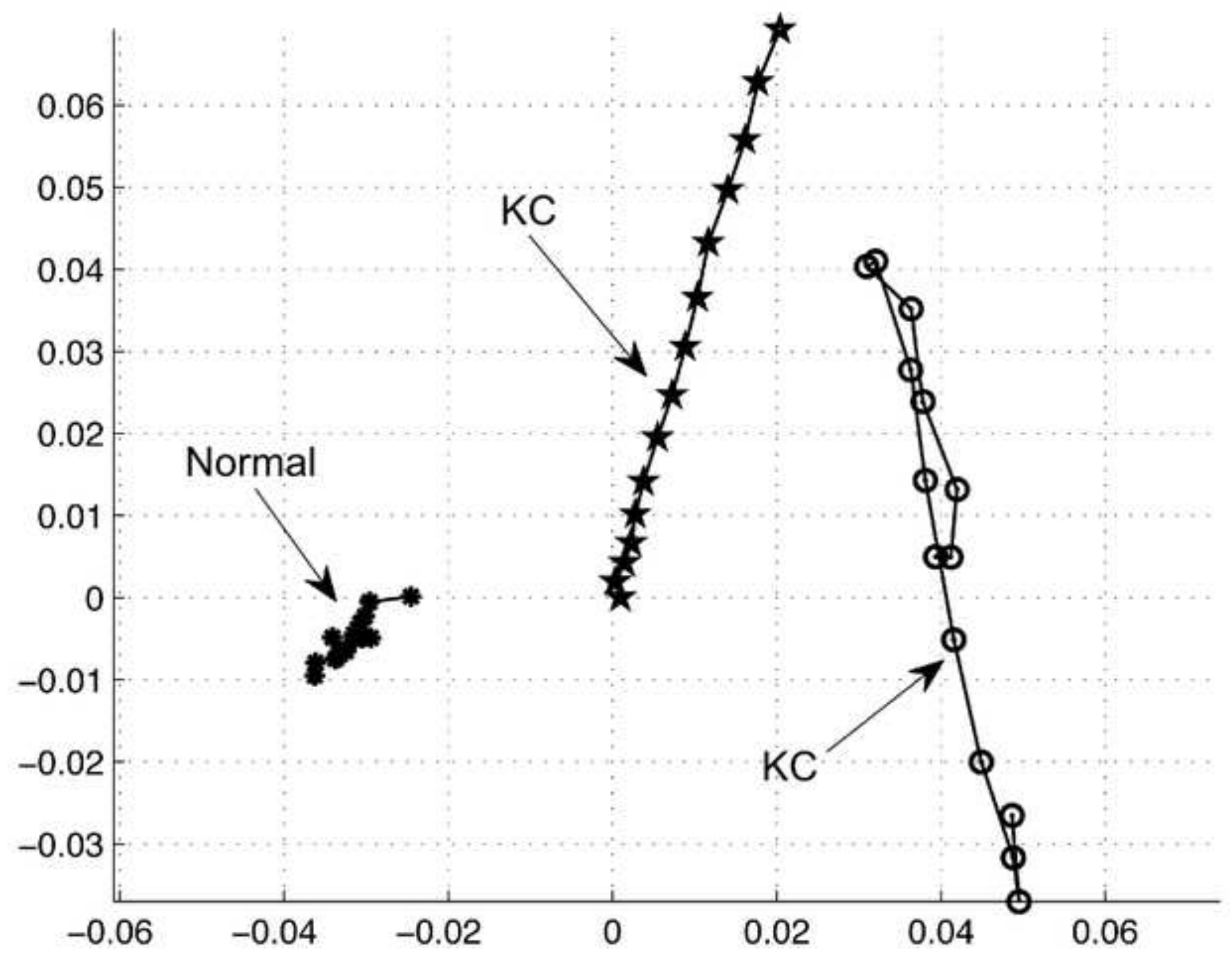




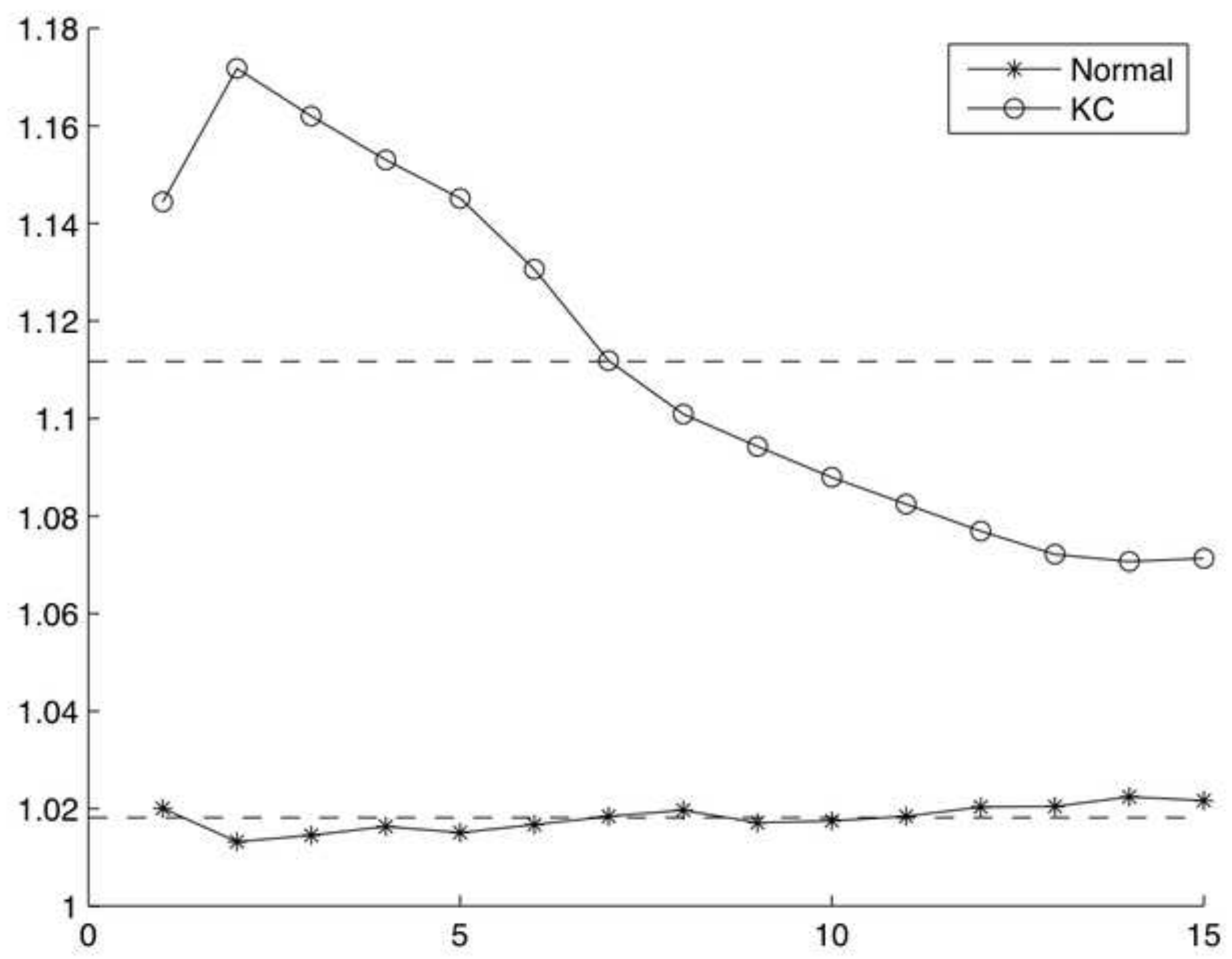




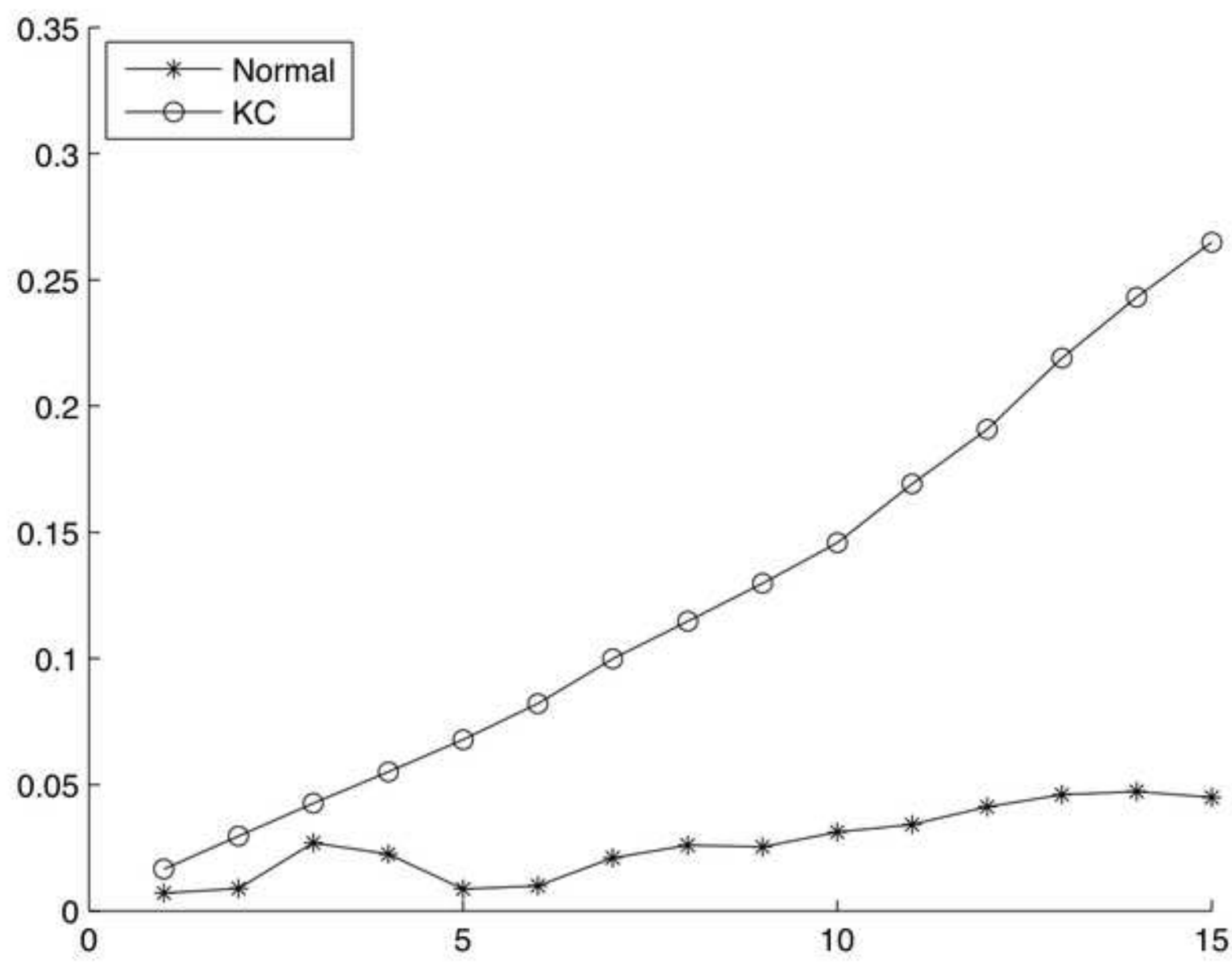




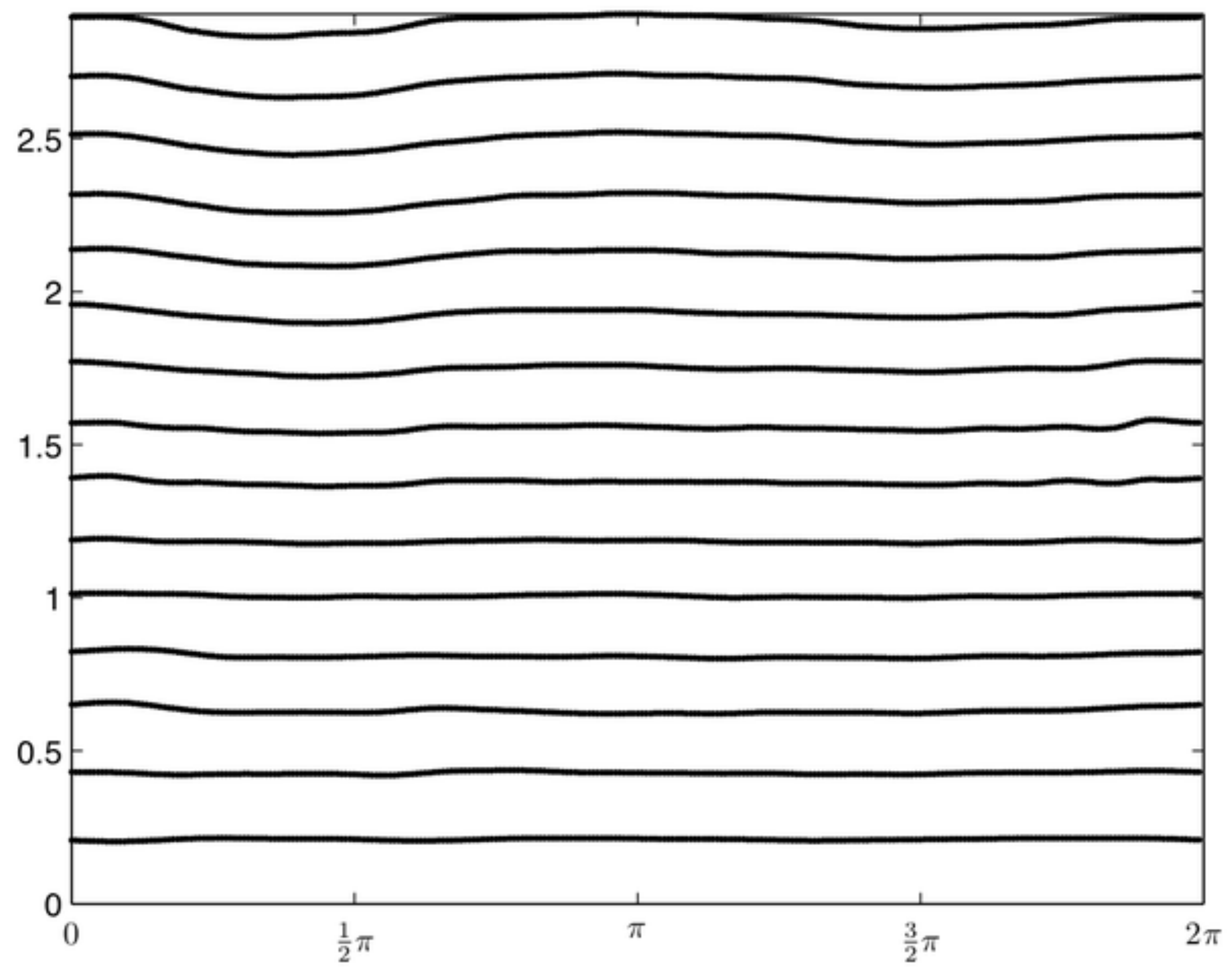


Click here to download high resolution image

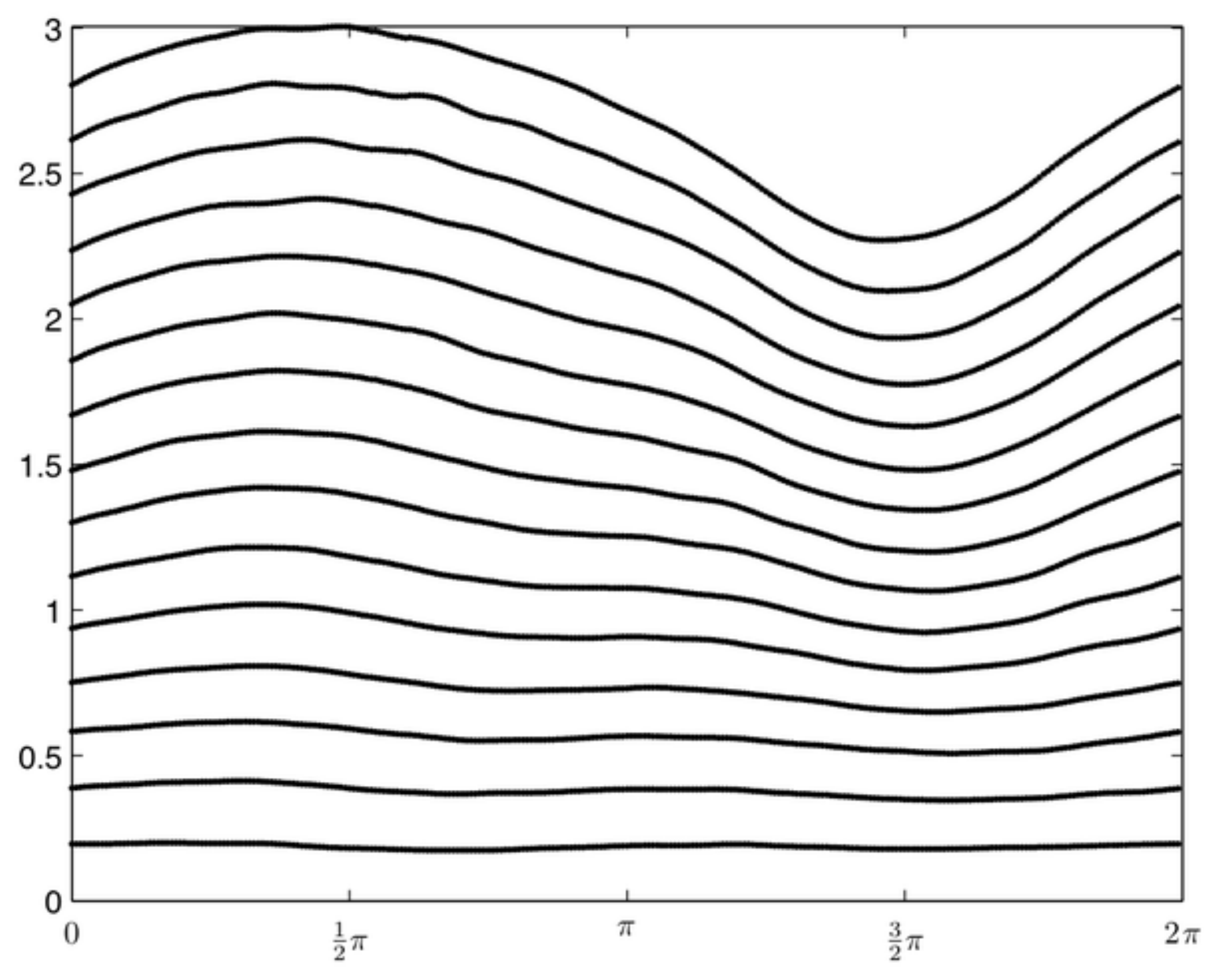

C 


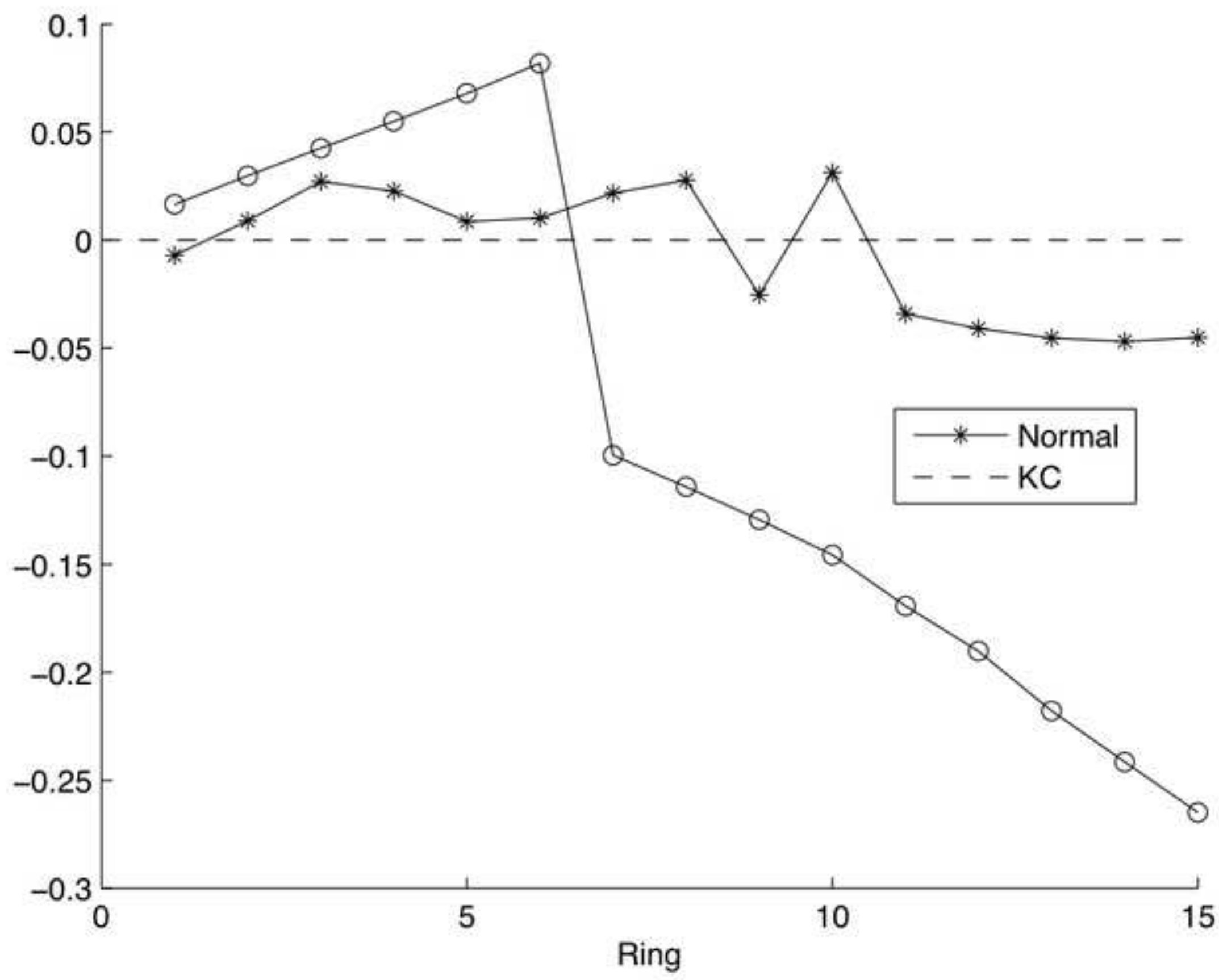




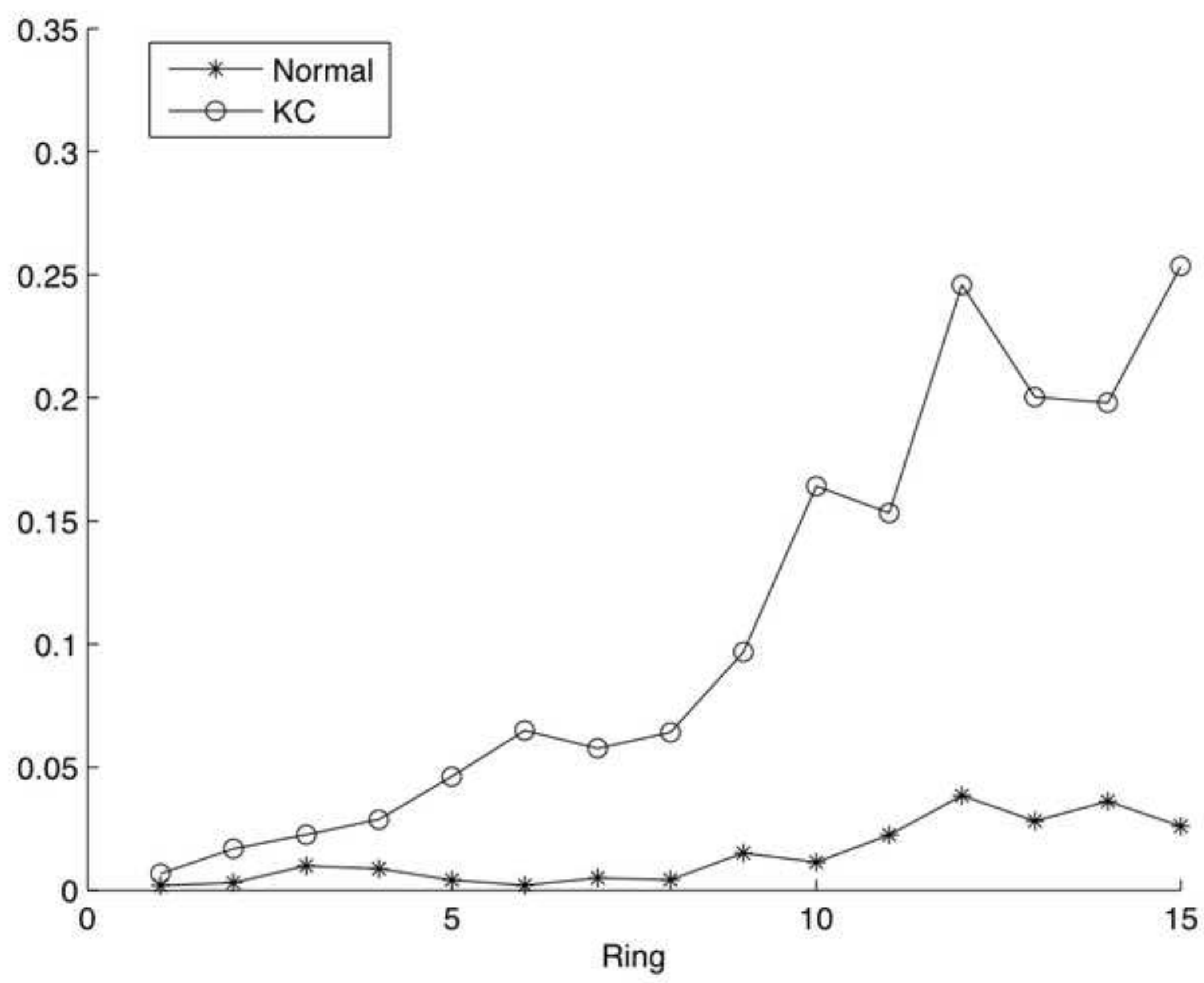


Click here to download high resolution image

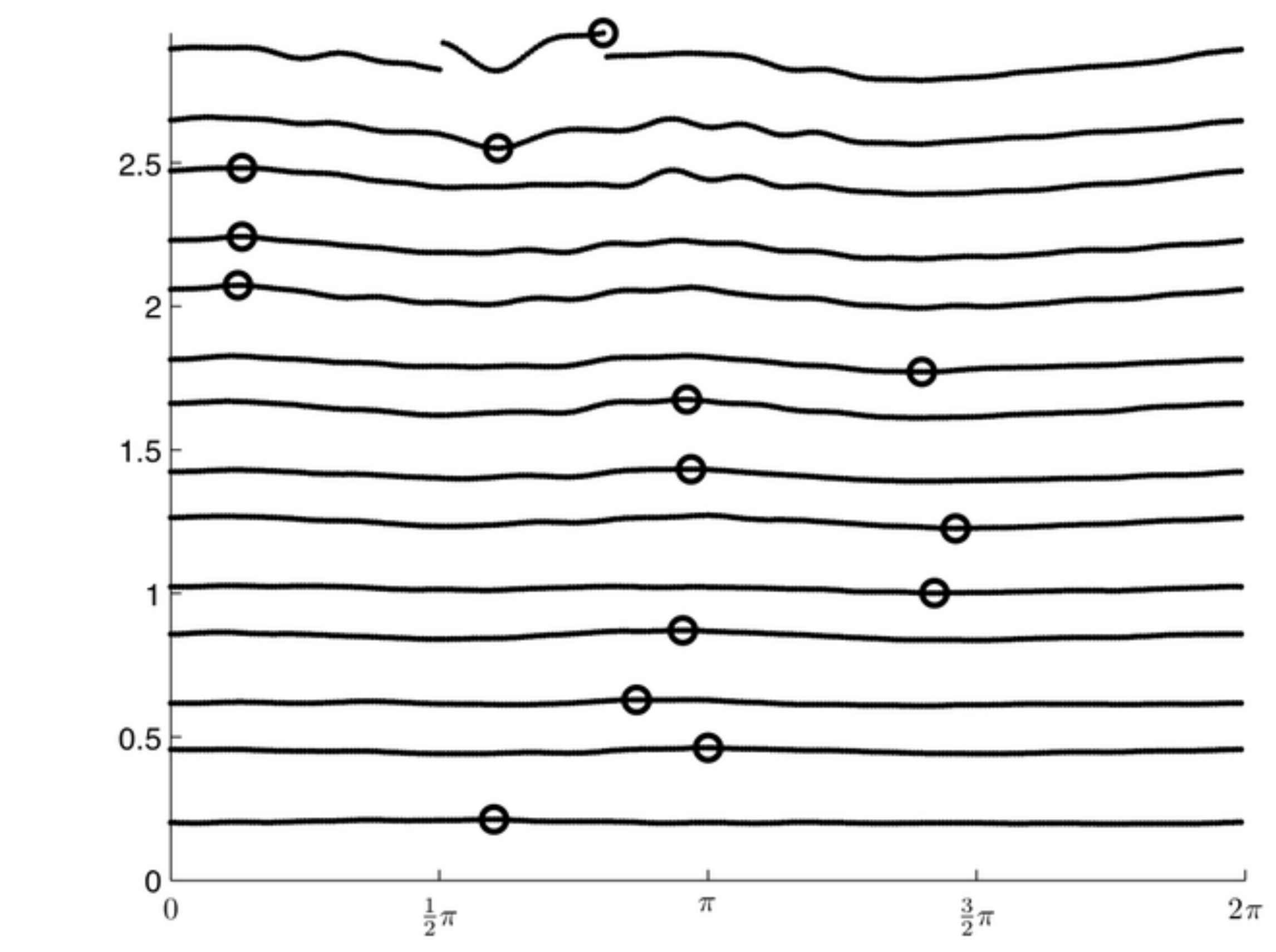


Click here to download high resolution image

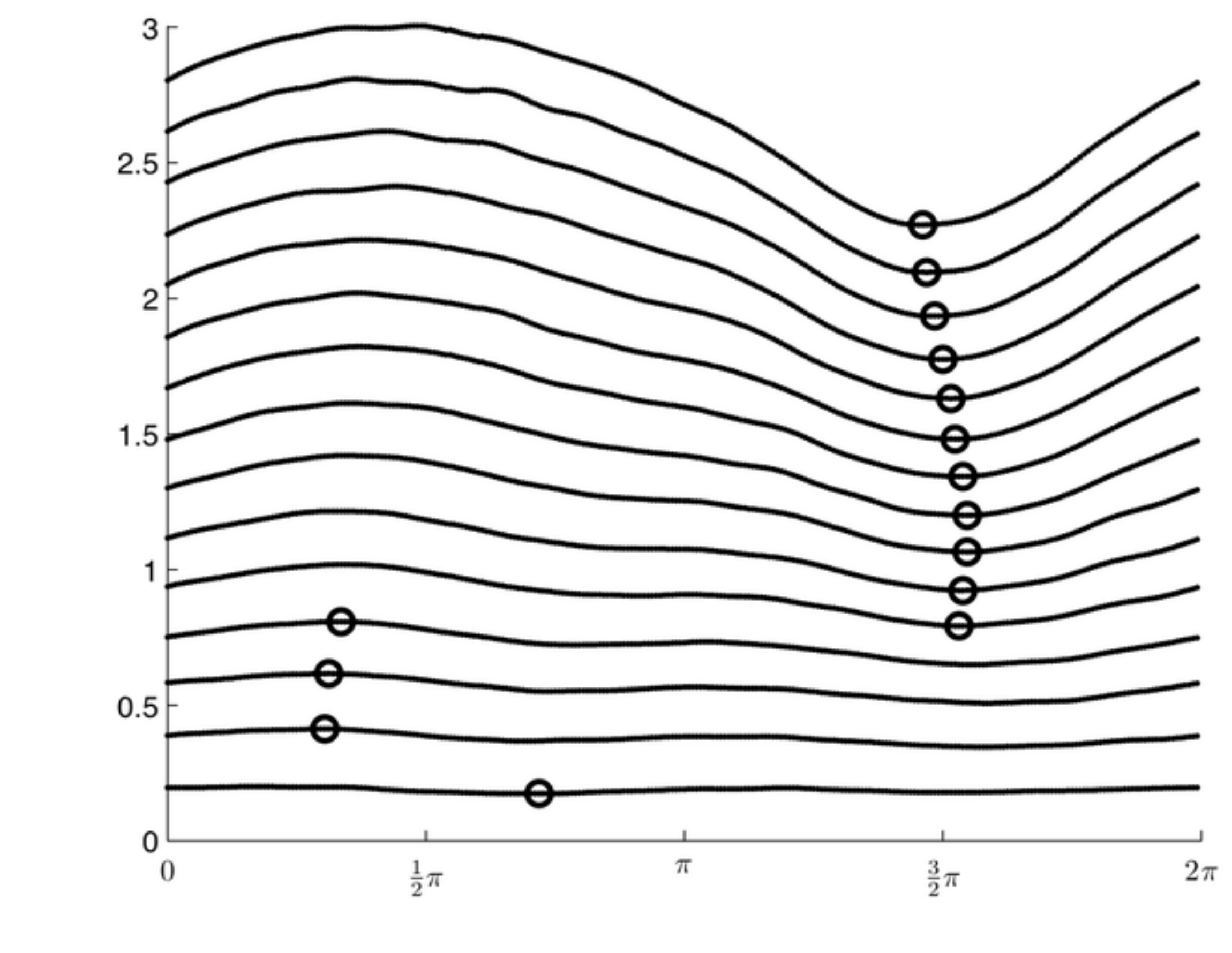

(d)

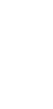


Figure
Click here to download high resolution image

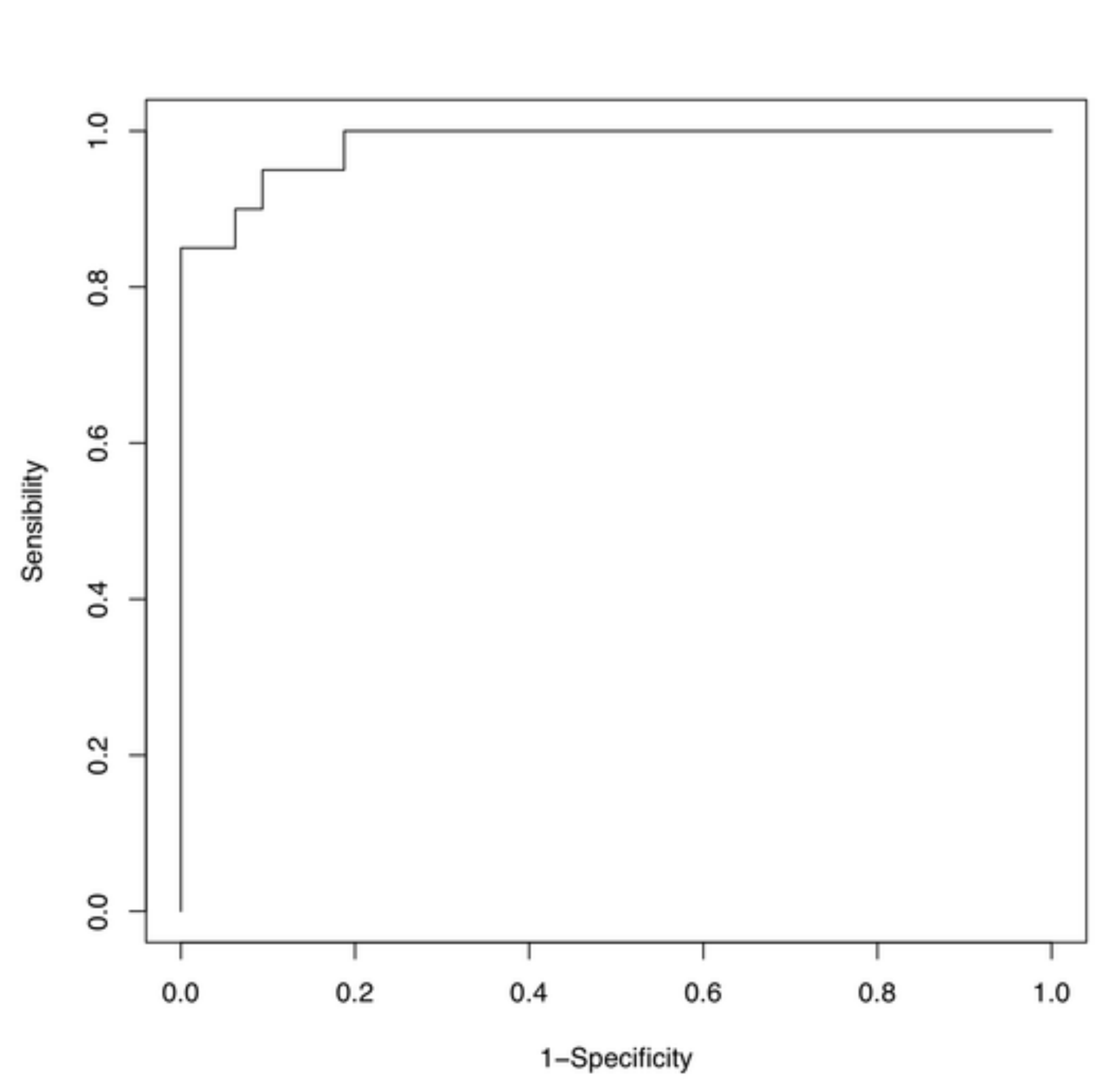

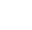

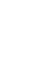


Figure
Click here to download high resolution image

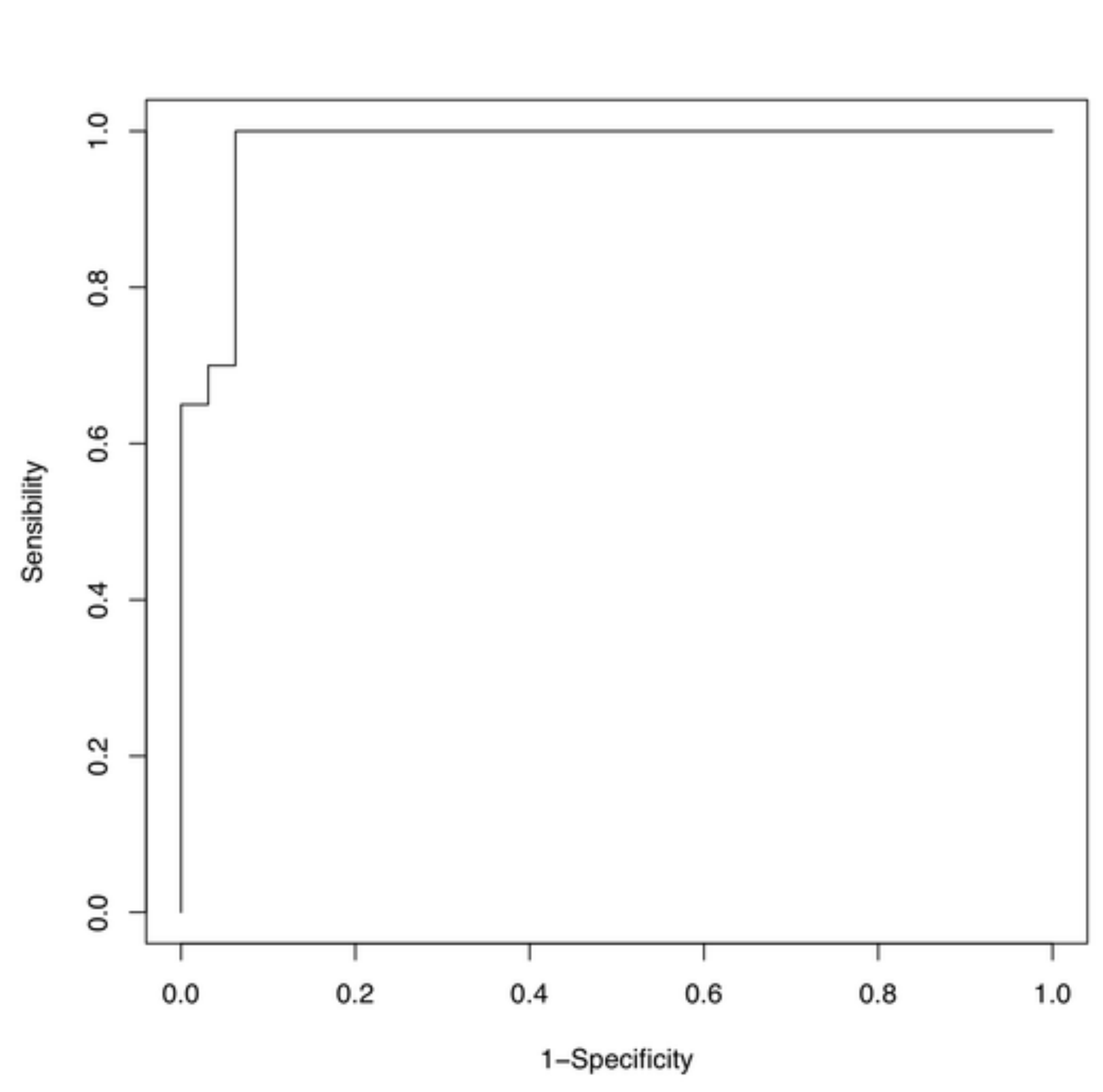

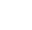

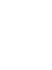


Figure
Click here to download high resolution image

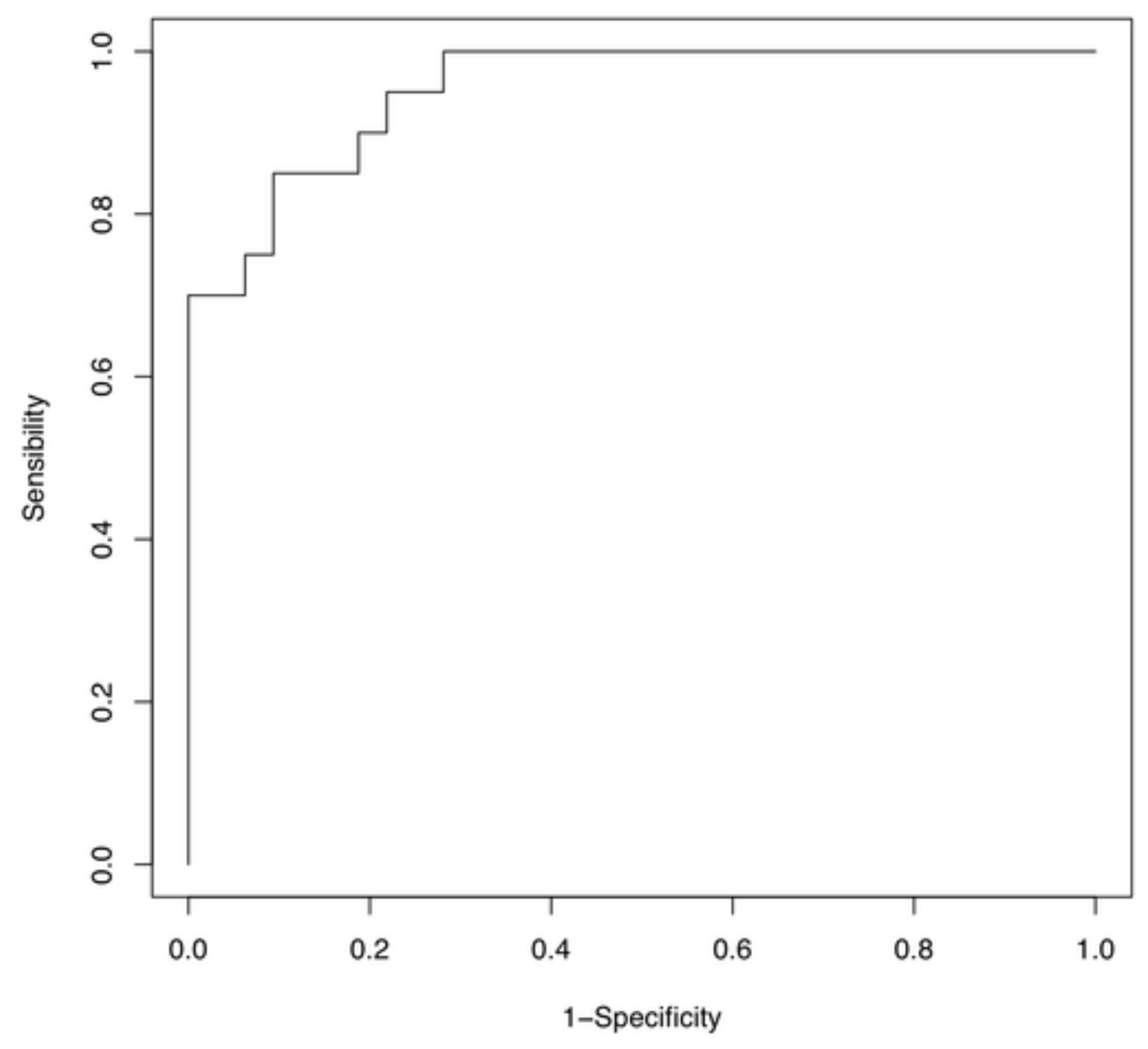


Figure
Click here to download high resolution image

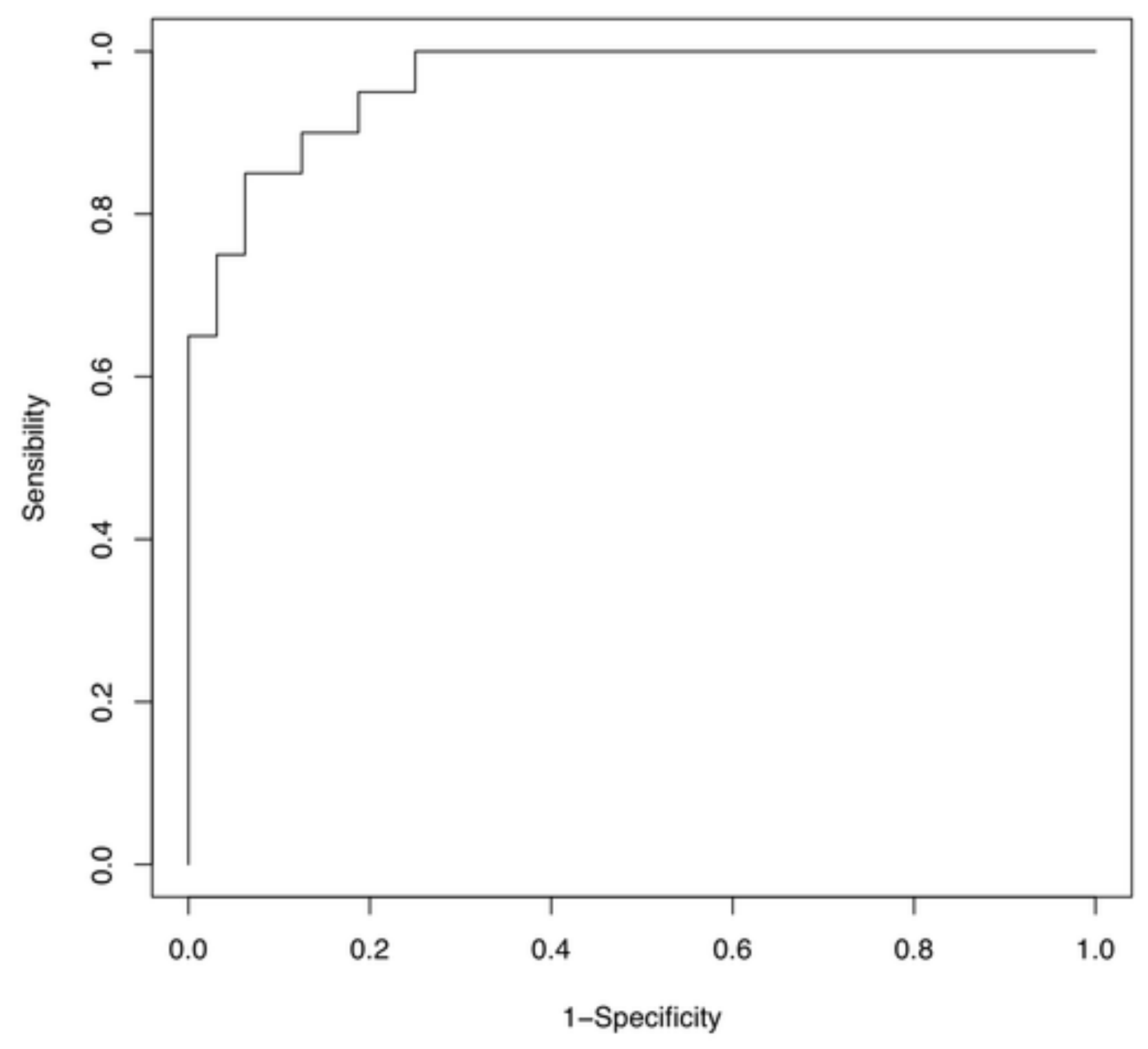


Click here to download high resolution image

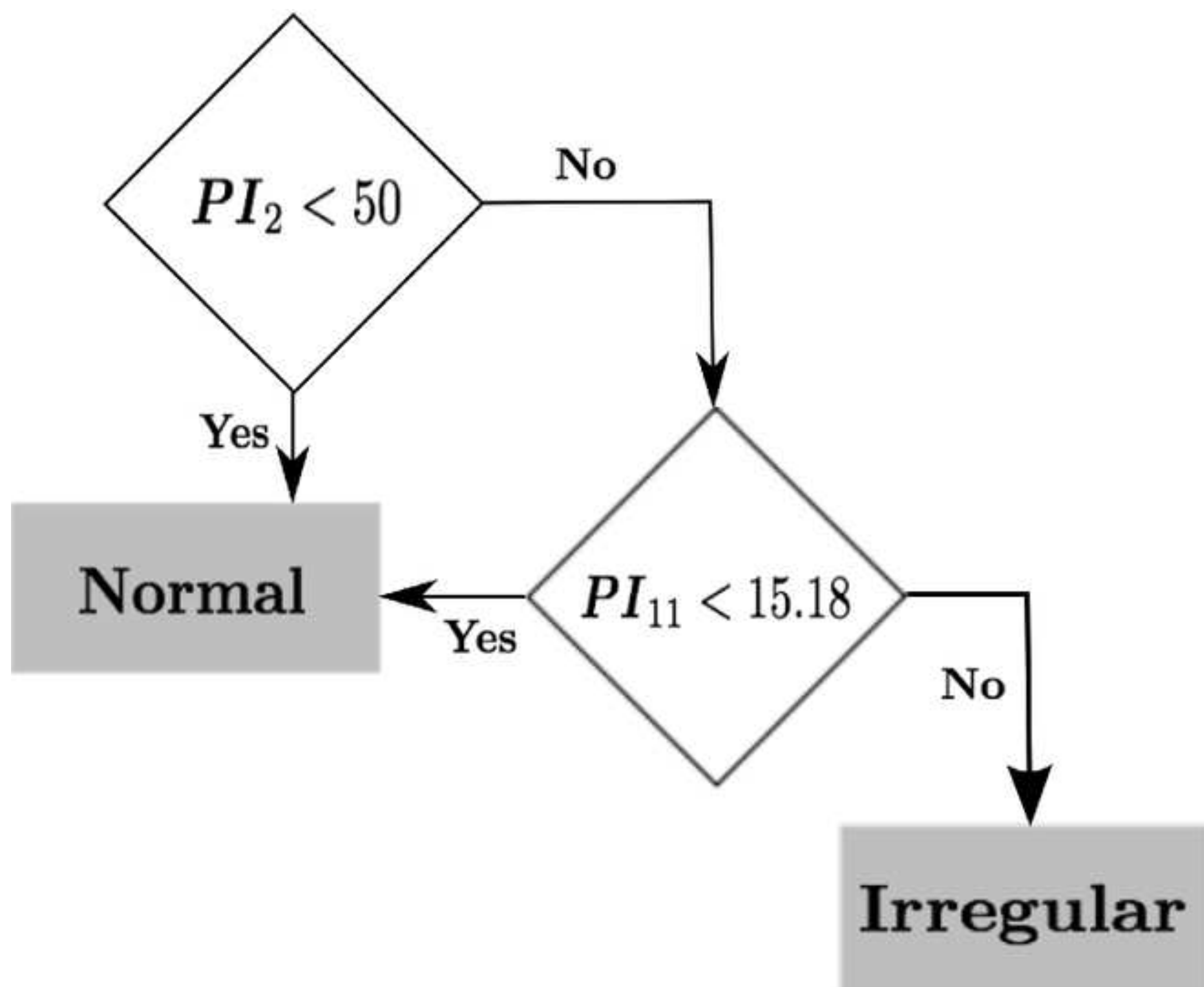


Figure
Click here to download high resolution image

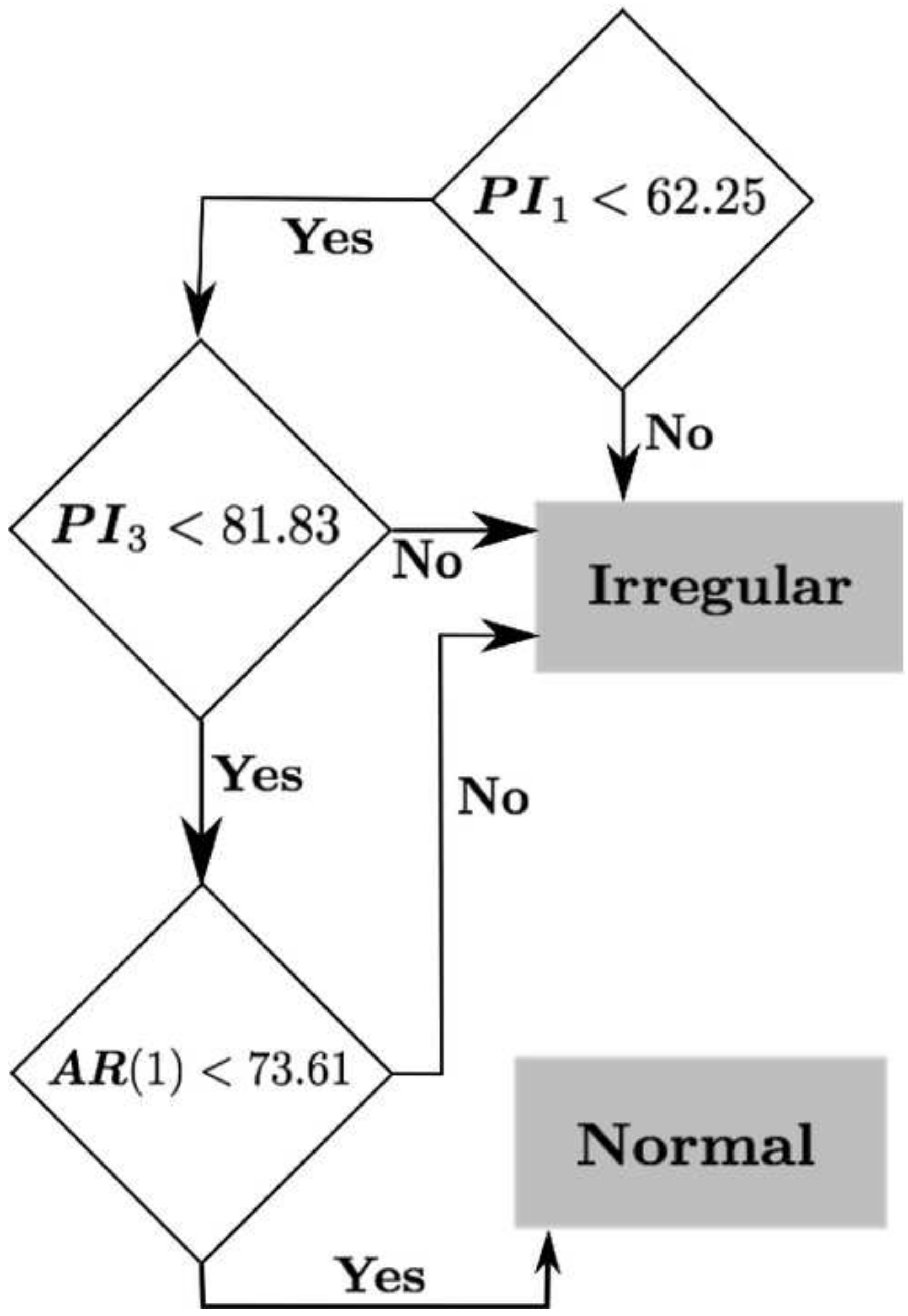




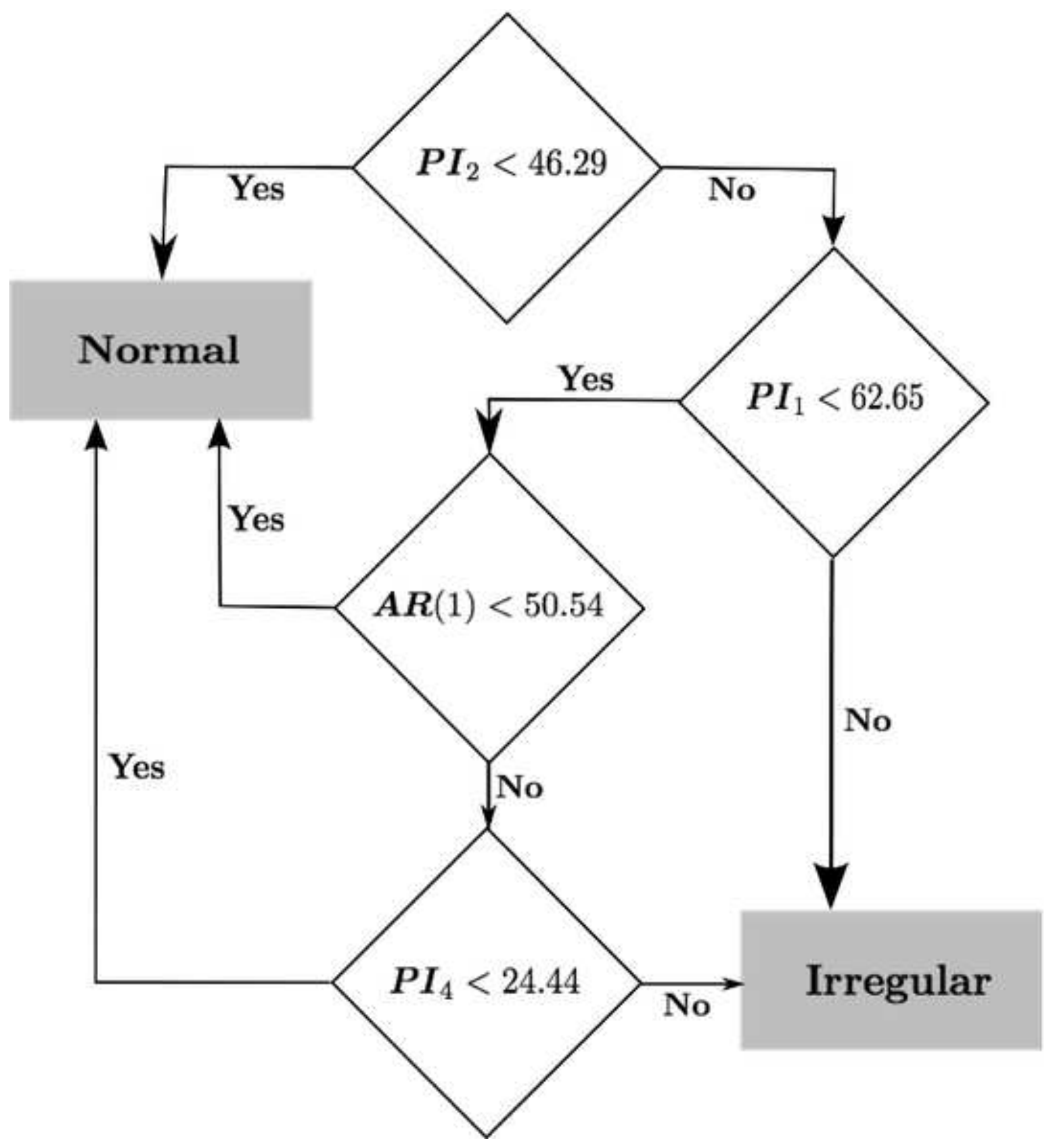

